STRUCTURAL BIOLOGY COMMUNICATIONS

ISSN 2053-230X
Received 8 November 2019

Accepted 3 February 2020

Edited by M. W. Bowler, European Molecular Biology Laboratory, France

Keywords: omalizumab; allergy; Fab; immunoglobulin E; antibody; protein engineering; X-ray crystallography.

PDB references: omalizumab Fab, crystal form I, 6tcm; crystal form II, 6tcn; Leu158Pro lightchain mutant, crystal form I, 6tco; crystal form II, 6tcp; Ser81Arg and Gln83Arg light-chain mutant, 6tcq; Ser81Arg, Gln83Arg and Leu158Pro light-chain mutant, 6tcr; omalizumab scFv, 6tcs

Supporting information: this article has supporting information at journals.iucr.org/f

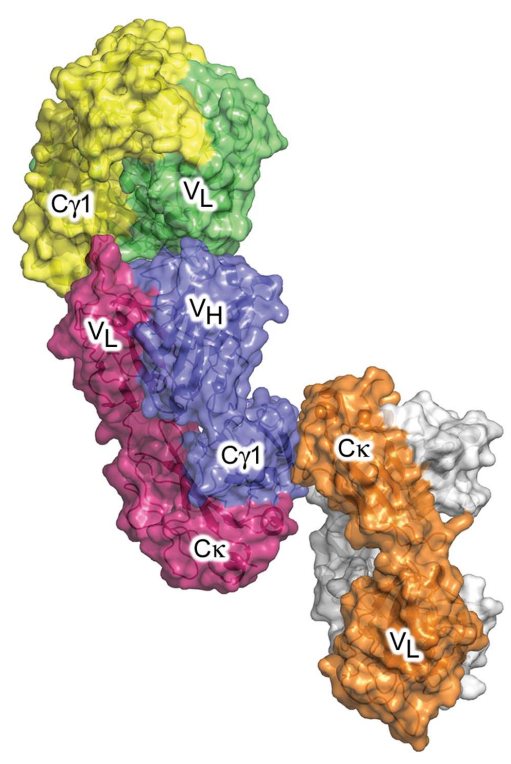

OPEN $\odot$ ACCESS

\section{Engineering the Fab fragment of the anti-IgE omalizumab to prevent Fab crystallization and permit IgE-Fc complex crystallization}

\author{
Alkistis N. Mitropoulou, ${ }^{\text {a,b }}$ Tom Ceska, ${ }^{\mathrm{c}}$ James T. Heads, ${ }^{\mathrm{c}}$ Andrew J. Beavil,, \\ Alistair J. Henry, ${ }^{c}$ James M. McDonnell, ${ }^{\mathrm{a}, \mathrm{b} *}$ Brian J. Sutton ${ }^{\mathrm{a}, \mathrm{b} *}$ and Anna M. \\ Davies $^{\mathrm{a}, \mathrm{b} *}$
}

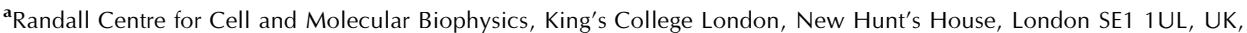
${ }^{\mathbf{b}}$ Medical Research Council and Asthma UK Centre in Allergic Mechanisms of Asthma, London, UK, and ${ }^{\mathbf{c}}$ UCB Celltech, 208 Bath Road, Slough SL1 3WE, UK. *Correspondence e-mail: james.mcdonnell@kcl.ac.uk, brian.sutton@kcl.ac.uk, anna.davies@kcl.ac.uk
\end{abstract}

Immunoglobulin E (IgE) plays a central role in the allergic response, in which cross-linking of allergen by $\mathrm{Fc} \varepsilon \mathrm{RI}$-bound IgE triggers mast cell and basophil degranulation and the release of inflammatory mediators. The high-affinity interaction between $\mathrm{IgE}$ and $\mathrm{Fc} \varepsilon \mathrm{RI}$ is a long-standing target for therapeutic intervention in allergic disease. Omalizumab is a clinically approved anti-IgE monoclonal antibody that binds to free IgE, also with high affinity, preventing its interaction with $\mathrm{Fc} \varepsilon \mathrm{RI}$. All attempts to crystallize the pre-formed complex between the omalizumab Fab and the Fc region of $\operatorname{IgE}(\operatorname{IgE}-\mathrm{Fc})$, to understand the structural basis for its mechanism of action, surprisingly failed. Instead, the Fab alone selectively crystallized in different crystal forms, but their structures revealed intermolecular Fab/Fab interactions that were clearly strong enough to disrupt the Fab/IgE-Fc complexes. Some of these interactions were common to other Fab crystal structures. Mutations were therefore designed to disrupt two recurring packing interactions observed in the omalizumab Fab crystal structures without interfering with the ability of the omalizumab Fab to recognize IgE-Fc; this led to the successful crystallization and subsequent structure determination of the Fab/IgE-Fc complex. The mutagenesis strategy adopted to achieve this result is applicable to other intractable Fab/antigen complexes or systems in which Fabs are used as crystallization chaperones.

\section{Introduction}

Immunoglobulin $\mathrm{E}(\mathrm{IgE})$ plays a central role in allergic disease through the interaction between its $\mathrm{Fc}$ region $(\mathrm{IgE}-\mathrm{Fc})$ and the $\mathrm{F} c \varepsilon \mathrm{RI}$ receptor, in which cross-linking of Fc $\varepsilon \mathrm{RI}$-bound IgE by allergen triggers mast cell and basophil degranulation, with the release of inflammatory mediators (Gould \& Sutton, 2008).

IgE-Fc, comprising two identical disulfide-linked chains of $\mathrm{C} \varepsilon 2, \mathrm{C} \varepsilon 3$ and $\mathrm{C} \varepsilon 4$ domains, adopts a bent conformation in solution (Beavil et al., 1995; Davis et al., 1990; Holowka \& Baird, 1983; Holowka et al., 1985; Hunt et al., 2012; Zheng et al., 1991, 1992). In the crystal structure of unbound IgE-Fc, the Fc region is acutely bent: the $(\mathrm{C} \varepsilon 2)_{2}$ domain pair folds back against the $\mathrm{C} \varepsilon 3$ and $\mathrm{C} \varepsilon 4$ domains, with an angle of $62^{\circ}$ between the local twofold axes of the $\mathrm{C} \varepsilon 2$ and $\mathrm{C} \varepsilon 4$ domain pairs (Doré et al., 2017; Holdom et al., 2011; Wan et al., 2002). The Fc $\varepsilon 3-4$ region, comprising only the $\mathrm{C} \varepsilon 3$ and $\mathrm{C} \varepsilon 4$ domains, is conformationally flexible, and the $\mathrm{C} \varepsilon 3$ domains can adopt a variety of positions relative to one another, from 'closed' to ‘open' (Chen et al., 2018; Cohen et al., 2014; Davies et al., 2017; 
Dhaliwal et al., 2012, 2014, 2017; Doré et al., 2017; Drinkwater et al., 2014; Garman et al., 2000; Holdom et al., 2011; Jabs et al., 2018; Wan et al., 2002; Wurzburg \& Jardetzky, 2009; Wurzburg et al., 2000; Yuan et al., 2013), a property associated with the mutually exclusive, allosteric regulation of binding to Fce RI and the second principal receptor for IgE, CD23 (Borthakur et al., 2012; Dhaliwal et al., 2012). The C $\varepsilon 3$ domains adopt an open conformation, and IgE-Fc becomes more acutely bent, when in complex with Fce RI (Garman et al., 2000; Holdom et al., 2011; Hunt et al., 2012), while CD23 binds when the Ce3 domains adopt a closed conformation (Dhaliwal et al., 2012, 2014, 2017; Yuan et al., 2013). The potential for more extreme flexibility in IgE-Fc was first revealed when a fully extended, linear structure, involving a $\sim 120^{\circ}$ unbending of the $(\mathrm{C} \varepsilon 2)_{2}$ domain pair relative to the Fce3-4 region, was captured by an anti-IgE Fab (Drinkwater et al., 2014). Molecular dynamics simulations have also revealed that IgE-Fc can adopt relatively stable, more extended conformations, between the two extremes of acutely bent and fully extended (Drinkwater et al., 2014).

The high-affinity interaction between $\operatorname{IgE}$ and $\mathrm{Fc} \varepsilon \mathrm{RI}$ is a long-standing target in the development of treatments for allergic disease (Holgate, 2014). Omalizumab is an anti-IgE therapeutic monoclonal IgG1 antibody that inhibits the interaction of $\operatorname{IgE}$ with $\mathrm{Fc} \varepsilon \mathrm{RI}$ and is approved for the treatment of moderate-to-severe persistent allergic asthma and chronic idiopathic urticaria (Holgate et al., 2005; Sussman et al., 2014). Although the binding site for omalizumab had previously been mapped to the $\mathrm{C} \varepsilon 3$ domain (Zheng et al., 2008), and omalizumab was known to bind to a partially bent IgE-Fc conformation (Hunt et al., 2012), the structural basis for its mechanism of action was poorly understood until only recently.

We, and others (Jensen et al., 2015), had attempted to crystallize the complex between the omalizumab Fab and IgE-Fc. However, despite extensive efforts, our crystallization trials of pre-formed omalizumab Fab/IgE-Fc and Fce3-4 complexes only resulted in selective crystallization of the Fab. The structure of the omalizumab Fab in complex with the Fce3-4 region of IgE-Fc has been reported, which revealed details of the omalizumab epitope on the $\mathrm{C} \varepsilon 3$ domain (Pennington et al., 2016). However, this Fce3-4 molecule lacked the $(\mathrm{C} \varepsilon 2)_{2}$ domain pair and was conformationally constrained by an engineered disulfide bond that locked the $\mathrm{C} \varepsilon 3$ domains into a closed conformation (Pennington et al., 2016). Given the flexible nature of the Fc\&3-4 region, and the potential for extreme flexibility in IgE-Fc, which additionally contains the $(\mathrm{C} \varepsilon 2)_{2}$ domain pair, this structure could thus provide only limited mechanistic insights.

We designed a mutagenesis strategy to disrupt the packing interactions observed in omalizumab Fab crystal structures, without affecting the antigen-binding CDRs, with the aim of crystallizing the complex between the omalizumab Fab and IgE-Fc. The strategy first involved creating a point mutation in a short segment of $\beta$-strand structure found in the $\mathrm{C} \kappa$ domain $\mathrm{CD}$ loop, followed by two point mutations in the $\mathrm{V}_{\mathrm{L}}$ domain EF loop.
One omalizumab-derived Fab, termed FabXol3, which contains three point mutations in the light chain, subsequently enabled us to solve the $3.7 \AA$ resolution crystal structure of the complex with $\mathrm{IgE}-\mathrm{Fc}$, revealing that omalizumab inhibits binding to FceRI allosterically (Davies et al., 2017). In this complex, IgE-Fc adopts a partially bent conformation, and the C $\varepsilon 3$ domains adopt a markedly open conformation, more open than that seen in any other crystal structure thus far.

Here, we report the structural basis and rationale for this mutagenesis strategy. Such an approach could inform the design and structure determination of other Fabs in complex with their target proteins in cases where the pre-formed complex is disrupted by the selective crystallization of one partner, in particular the Fab.

\section{Materials and methods}

\subsection{Macromolecule production}

IgE-Fc, Fce3-4, FabXol, FabXol2, FabXol3 and scFvXol proteins were produced using previously described methods (Davies et al., 2017; Dhaliwal et al., 2012; Drinkwater et al., 2014; Weatherill et al., 2012; Young et al., 1995). Omalizumab was purchased from Novartis Europharm Ltd.

\subsection{Crystallization}

All crystals were grown at $18^{\circ} \mathrm{C}$ using the sitting-drop vapor-diffusion method in MRC 96-well plates. FabXol ${ }^{1}$ and $\mathrm{FabXol}^{2}$ (omalizumab Fab) crystals were grown from unsuccessful crystallization trials of the FabXol/IgE-Fc and FabXol/Fce3-4 complexes. For the $\mathrm{FabXol}^{1}$ and $\mathrm{FabXol}^{2}$ structures reported here, the 2:1 complex between FabXol and Fc\&3-4 was purified by size-exclusion chromatography, bufferexchanged into $25 \mathrm{~m} M$ Tris- $\mathrm{HCl} \mathrm{pH} 7.5,20 \mathrm{mM} \mathrm{NaCl}$ and concentrated to $18.8 \mathrm{mg} \mathrm{ml}^{-1}$. FabXol ${ }^{1}$ crystals were grown in $0.085 M$ Tris $\mathrm{pH} 8.5,42.5 \%(v / v)$ MPD, $15 \%(v / v)$ glycerol, $0.17 M$ ammonium phosphate and were cryoprotected with the mother liquor. FabXol ${ }^{2}$ crystals were grown in $0.1 \mathrm{M}$ phosphate-citrate $\mathrm{pH} 4.2,20 \%(w / v)$ PEG 1000, $0.2 M$ lithium sulfate and were cryoprotected with $0.1 M$ sodium acetate $\mathrm{pH}$ 4.6, $25 \%(w / v)$ PEG $4000,18 \%(v / v)$ ethylene glycol. For both crystals, a reservoir volume of $50 \mu \mathrm{l}$ was used and the drops consisted of $100 \mathrm{nl}$ protein solution and $200 \mathrm{nl}$ reservoir solution.

FabXol1 ${ }^{1}$ and FabXol1 ${ }^{2}$ (omalizumab-derived Leu158Pro light-chain mutant Fab) crystals were grown from unsuccessful crystallization trials of the FabXol1/IgE-Fc complex. The 2:1 complex between FabXol1 and IgE-Fc was purified by sizeexclusion chromatography, buffer-exchanged into $0.25 \mathrm{M}$ Tris$\mathrm{HCl} \mathrm{pH} \mathrm{7.5,} 0.2 \mathrm{M} \mathrm{NaCl}$ and concentrated to $18.8 \mathrm{mg} \mathrm{ml}^{-1}$. FabXol $1^{1}$ crystals were grown in 20\%(w/v) PEG 3350, $0.2 \mathrm{M}$ sodium sulfate and were cryoprotected with $20 \%(w / v)$ PEG 3350, $0.2 \mathrm{M}$ magnesium sulfate, $18 \%(v / v)$ ethylene glycol. FabXol1 ${ }^{2}$ crystals were grown in $20 \%(w / v)$ PEG 4000, $0.2 M$ magnesium sulfate, $10 \%(v / v)$ glycerol and were cryoprotected in $20 \%(w / v)$ PEG 4000, $0.2 M$ magnesium sulfate, $18 \%(v / v)$ glycerol. For both crystals, a reservoir volume of $50 \mu \mathrm{l}$ was 
Table 1

Data-processing and refinement statistics for $\mathrm{FabXol}^{1}, \mathrm{FabXol}^{2}$ and scFvXol.

Values in parentheses are for the outer shell.

\begin{tabular}{|c|c|c|c|}
\hline & FabXol $^{1}$ & $\mathrm{FabXol}^{2}$ & scFvXol \\
\hline \multicolumn{4}{|l|}{ Data processing } \\
\hline Space group & $P 2_{1} 2_{1} 2_{1}$ & $P 12_{1} 1$ & $P 3_{1} 21$ \\
\hline$a, b, c(\AA)$ & $\begin{array}{c}\text { 65.38, } 73.56 \\
141.10\end{array}$ & $\begin{array}{c}85.29,73.57 \\
87.10\end{array}$ & $\begin{array}{c}\text { 73.91, } 73.91 \\
117.80\end{array}$ \\
\hline$\beta\left(^{\circ}\right)$ & & 116.58 & \\
\hline Resolution $(\AA)$ & $\begin{array}{l}65.38-1.85 \\
\quad(1.89-1.85)\end{array}$ & $\begin{array}{l}77.89-2.30 \\
\quad(2.42-2.30)\end{array}$ & $\begin{array}{l}64.01-2.30 \\
\quad(2.38-2.30)\end{array}$ \\
\hline Completeness (\%) & $99.9(99.9)$ & $99.5(96.9)$ & $99.8(99.9)$ \\
\hline Multiplicity & $7.2(6.9)$ & $3.7(3.2)$ & $5.5(5.2)$ \\
\hline Mean $I / \sigma(I)$ & $4.3(1.7)$ & $9.0(1.8)$ & $6.2(2.5)$ \\
\hline $\mathrm{CC}_{1 / 2}$ & $0.99(0.413)$ & $0.993(0.631)$ & $0.974(0.597)$ \\
\hline$R_{\text {p.i.m. }}$ & $0.078(1.101)$ & $0.060(0.454)$ & $0.121(1.064)$ \\
\hline$R_{\text {merge }}$ & $0.192(2.641)$ & $0.099(0.674)$ & $0.259(2.248)$ \\
\hline Wilson $B$ factor $\left(\AA^{2}\right)$ & 22.8 & 44.0 & 24.6 \\
\hline \multicolumn{4}{|l|}{ Refinement } \\
\hline$R_{\text {work }} / R_{\text {free }} \dagger(\%)$ & $16.77 / 19.03$ & $18.78 / 22.58$ & $17.80 / 20.62$ \\
\hline No. of reflections & 57943 & 42804 & 16902 \\
\hline \multicolumn{4}{|l|}{ R.m.s. deviations } \\
\hline Bond lengths $(\AA)$ & 0.014 & 0.002 & 0.004 \\
\hline Bond angles $\left({ }^{\circ}\right)$ & 1.334 & 0.535 & 0.683 \\
\hline Coordinate error $(\AA ̊)$ & 0.18 & 0.30 & 0.20 \\
\hline \multicolumn{4}{|l|}{ No. of atoms } \\
\hline Protein & 3357 & 6468 & 1751 \\
\hline Solvent & 314 & 246 & 78 \\
\hline Other & $70 \ddagger$ & $62 \S$ & 14 ब \\
\hline \multicolumn{4}{|l|}{ Average $B$ factor $\left(\AA^{2}\right)$} \\
\hline Protein & 27.81 & 47.55 & 33.68 \\
\hline Solvent & 40.07 & 41.26 & 38.72 \\
\hline Other & $51.82 \ddagger$ & $62.02 \S$ & 53.20 ๑ \\
\hline \multicolumn{4}{|l|}{ Ramachandran plot } \\
\hline Favored (\%) & 97.79 & 97.07 & 96.98 \\
\hline Allowed (\%) & 2.21 & 2.82 & 3.02 \\
\hline
\end{tabular}

$\dagger$ The $R_{\text {free }}$ set comprises $5 \%$ of the reflections. $\ddagger 2$-Methyl-2,4-pentanediol, glycerol and phosphate. $\S$ Ethylene glycol, polyethylene glycol, Tris and sulfate. $\uparrow$ Polyethylene glycol.

used and the drops consisted of $100 \mathrm{nl}$ protein solution and $200 \mathrm{nl}$ reservoir solution.

FabXol2 (omalizumab-derived Ser81Arg, Gln83Arg lightchain mutant $\mathrm{Fab}$ ) was buffer-exchanged into $0.1 \mathrm{M}$ Tris- $\mathrm{HCl}$ $\mathrm{pH} 8.5,0.05 \mathrm{M} \mathrm{NaCl}$ and concentrated to $3 \mathrm{mg} \mathrm{ml}^{-1}$. FabXol2 crystals were grown in $0.1 M$ HEPES $\mathrm{pH} 7,20 \%(w / v)$ PEG 4000 and were cryoprotected with $12 \%(v / v)$ PEG 400 , $17 \%(v / v)$ glycerol; a reservoir volume of $100 \mu \mathrm{l}$ was used and the drops consisted of $200 \mathrm{nl}$ protein solution and $100 \mathrm{nl}$ reservoir solution. FabXol3 (omalizumab-derived Ser81Arg, Gln83Arg, Leu158Pro light-chain mutant Fab) was purified in phosphate-buffered saline (PBS), concentrated to $15 \mathrm{mg} \mathrm{ml}^{-1}$ and then diluted to $5 \mathrm{mg} \mathrm{ml}^{-1}$ with $0.1 M$ Tris $\mathrm{pH} 8.5$. FabXol3 crystals were grown in $0.1 M$ HEPES pH 7, 20\%(w/v) PEG 4000, $0.15 M$ ammonium sulfate and were cryoprotected with $0.1 M$ HEPES $\mathrm{pH}$ 7.5, 20\%( $w / v)$ PEG 4000, $0.1 M$ ammonium sulfate, $15 \%(v / v)$ ethylene glycol; a reservoir volume of $50 \mu \mathrm{l}$ was used and the drops consisted of $100 \mathrm{nl}$ protein solution and $200 \mathrm{nl}$ reservoir solution. scFvXol was buffer-exchanged into $0.25 M$ Tris- $\mathrm{HCl} \mathrm{pH} \mathrm{8.5,} 0.2 \mathrm{M} \mathrm{NaCl}$ and concentrated to $3.9 \mathrm{mg} \mathrm{ml}^{-1}$. scFvXol crystals were grown in $0.1 M$ trisodium citrate $\mathrm{pH} 5.6,15 \%(w / v)$ PEG 4000, $0.2 M$ ammonium sulfate and were cryoprotected with $0.1 M$ trisodium citrate $\mathrm{pH}$ 5.6,
$30 \%(w / v)$ PEG 4000, $0.2 M$ ammonium sulfate; a reservoir volume of $100 \mu \mathrm{l}$ was used and the drops consisted of $100 \mathrm{nl}$ protein solution and $80 \mathrm{nl}$ reservoir solution.

\subsection{X-ray data collection, processing, structure} determination and refinement

Data were collected on beamlines I02, I03, I04, I04-1 and I24 at the Diamond Light Source, Harwell, UK. Data were integrated with $X D S$ (Kabsch, 2010) using the xia2 package (Winter, 2010) or with MOSFLM (Leslie \& Powell, 2007), and were scaled with AIMLESS (Evans \& Murshudov, 2013) or SCALA (Evans, 2006) from the CCP4 suite (Winn et al., 2011). Structures were solved by molecular replacement using MOLREP (Vagin \& Teplyakov, 2010) or Phaser (McCoy et al., 2007). Protein atoms from PDB entry 2fjf (Fuh et al., 2006) were used as a search model for the $\mathrm{FabXol}^{1}$ structure. Subsequent structures were solved using protein atoms $\left(\mathrm{V}_{\mathrm{H}}\right.$, $\mathrm{V}_{\mathrm{L}}, \mathrm{C} \kappa$ and $\mathrm{C} \gamma 1$ domains) from the $\mathrm{FabXol}^{1}$ structure as a search model, although the CDR residues were removed. The structures were initially refined with REFMAC (Murshudov et al., 2011) and subsequently with Phenix (Liebschner et al., 2019), and refinement was alternated with rounds of manual model building with Coot (Emsley et al., 2010). Model quality was assessed with MolProbity (Chen et al., 2010). Dataprocessing and refinement statistics are summarized in Tables 1 and 2. Interfaces were analyzed with PISA (Krissinel \& Henrick, 2007). Figures were produced with $P y M O L$.

\subsection{PDB references}

Coordinates and structure factors have been deposited in the Protein Data Bank with the following accession codes: $\mathrm{FabXol}^{1}, 6 \mathrm{tcm} ; \mathrm{FabXol}^{2}, 6 \mathrm{tcn} ; \mathrm{FabXol}^{1}{ }^{1}$, 6tco; FabXol1 ${ }^{2}, 6 \mathrm{tcp}$; FabXol2, 6tcq; FabXol3, 6tcr; scFvXol, 6tcs.

\subsection{Fluorescence-based thermal stability $\left(T_{\mathrm{m}}\right)$ measurement}

A thermal stability assay was performed using a QuantStudio 7 Real-Time PCR System (Thermo Fisher). $5 \mu \mathrm{l}$ of $30 \times$ SYPRO Orange Protein Gel Stain (Thermo Fisher), diluted from $5000 \times$ concentrate with PBS pH 7.4, was added to $45 \mu \mathrm{l}$ protein sample $\left(0.2 \mathrm{mg} \mathrm{ml}^{-1}\right.$ in PBS $\left.\mathrm{pH} 7.4\right)$ and mixed. $10 \mu \mathrm{l}$ of this solution was dispensed into an optical 384-well PCR plate. The PCR heating device was set at $20^{\circ} \mathrm{C}$ and increased to $99^{\circ} \mathrm{C}$ at a rate of $1.1^{\circ} \mathrm{C} \mathrm{min}{ }^{-1}$. A charge-coupled device was used to monitor fluorescence changes in the wells. Fluorescence intensity increases were plotted and the inflection point of the slope was used to generate apparent midpoint temperatures $\left(T_{\mathrm{m}}\right)$.

\subsection{Surface plasmon resonance}

Surface plasmon resonance binding experiments were performed using a Biacore T200 instrument (GE Healthcare). Intact omalizumab, the Fabs and $\mathrm{scFv}$ were immobilized at similar densities on CM5 sensor chips using an amine-coupling protocol according to the manufacturer's instructions (GE Healthcare). The following immobilization densities were used for these studies: omalizumab, 970 resonance units; 
Table 2

Data-processing and refinement statistics for FabXol1 ${ }^{1}$, FabXol1 ${ }^{2}$, FabXol2 and FabXol3.

Values in parentheses are for the outer shell.

\begin{tabular}{|c|c|c|c|c|}
\hline & FabXol1 ${ }^{1}$ & FabXol1 ${ }^{2}$ & FabXol2 & FabXol3 \\
\hline Space group & $C 222_{1}$ & $P 2_{1} 2_{1} 2_{1}$ & $P 2_{1} 2_{1} 2_{1}$ & $P 2_{1} 2_{1} 2_{1}$ \\
\hline$a, b, c(\AA)$ & $94.46,116.84,181.16$ & $80.11,162.04,164.43$ & $44.03,96.61,103.51$ & $43.72,96.25,103.30$ \\
\hline Completeness (\%) & $99.9(99.9)$ & $99.9(99.8)$ & $99.7(96.3)$ & $99.6(97.6)$ \\
\hline Multiplicity & $10.0(10.3)$ & $6.7(6.7)$ & $7.5(4.1)$ & $6.8(4.4)$ \\
\hline Mean $I / \sigma(I)$ & $15.9(1.7)$ & $8.9(1.9)$ & $7.5(1.7)$ & $14.8(2.7)$ \\
\hline $\mathrm{CC}_{1 / 2}$ & $0.999(0.596)$ & $0.984(0.562)$ & $0.990(0.608)$ & $0.997(0.832)$ \\
\hline Wilson $B$ factor $\left(\AA^{2}\right)$ & 26.8 & 13.2 & 17.4 & 11.2 \\
\hline \multicolumn{5}{|l|}{ Refinement } \\
\hline$R_{\text {work }} / R_{\text {free }} \dagger(\%)$ & $16.76 / 19.22$ & $21.23 / 23.88$ & $17.35 / 22.14$ & $16.60 / 18.36$ \\
\hline No. of reflections & 92661 & 74660 & 28385 & 77571 \\
\hline \multicolumn{5}{|l|}{ R.m.s. deviations } \\
\hline Bond lengths $(\AA)$ & 0.011 & 0.002 & 0.008 & 0.016 \\
\hline Bond angles $\left({ }^{\circ}\right)$ & 1.148 & 0.529 & 0.954 & 1.482 \\
\hline Other & $102 \ddagger$ & $128 \S$ & $6 \Phi$ & $51+\dagger$ \\
\hline \multicolumn{5}{|l|}{ Average $B$ factor $\left(\AA^{2}\right)$} \\
\hline Protein & 32.47 & 36.23 & 23.11 & 19.36 \\
\hline Solvent & 38.84 & 30.32 & 30.69 & 31.24 \\
\hline Other & $56.02 \ddagger$ & $61.11 \S$ & 45.81 ๑ & $34.92+\dagger$ \\
\hline \multicolumn{5}{|l|}{ Ramachandran plot } \\
\hline Favored (\%) & 97.62 & 97.12 & 97.70 & 98.22 \\
\hline Allowed (\%) & 2.38 & 2.88 & 2.30 & 1.78 \\
\hline
\end{tabular}

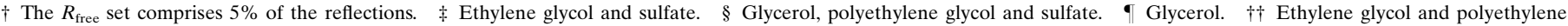
glycol.

Table 3

Nomenclature for the omalizumab-derived Fabs and scFv.

\begin{tabular}{|c|c|c|c|}
\hline Construct & Sequence & Structure & $\begin{array}{l}\text { No. of molecules in the } \\
\text { asymmetric unit }\end{array}$ \\
\hline FabXol & Wild type & FabXol $^{1}$ & $\begin{array}{l}\text { 1: } \mathrm{FabXol}^{1} \\
\text { 2. } \mathrm{FabXol}^{2 A} \mathrm{FabXol}^{2 B}\end{array}$ \\
\hline \multirow[t]{2}{*}{ FabXol1 } & \multirow[t]{2}{*}{ Leu158Pro $\dagger$} & $\begin{array}{l}\text { FabXol } \\
\text { FabXol1 }\end{array}$ & 2: FabXol1 ${ }^{1 A}, \mathrm{FabXol}^{1 B}$ \\
\hline & & FabXol1 ${ }^{2}$ & $\begin{array}{l}\text { 4: FabXol1 }{ }^{2 A}, \text { FabXol1 }{ }^{2 B}, \\
\text { FabXol1 }^{2 C}, \text { FabXol1 }\end{array}$ \\
\hline FabXol2 & Ser81Arg $\dagger$, Gln83Arg $\dagger$ & FabXol2 & 1: FabXol2 \\
\hline FabXol3 & $\begin{array}{l}\text { Ser81Arg } \dagger, \text { Gln83Arg } \dagger, \\
\text { Leu158Pro } \dagger\end{array}$ & FabXol3 & 1: FabXol3 \\
\hline scFvXol & Wild type $\ddagger$ & scFvXol & 1: scFvXol \\
\hline
\end{tabular}

$\dagger$ Mutation in the Fab light chain. $\ddagger$ The $\mathrm{V}_{\mathrm{L}}$ and $\mathrm{V}_{\mathrm{H}}$ domains are linked by a $\left(\mathrm{Gly}_{4} \mathrm{Ser}\right)_{4}$ linker.

FabXol, 200 resonance units; FabXol2, 270 resonance units; FabXol3, 210 resonance units; scFvXol, 250 resonance units. For binding studies, IgE-Fc, in a twofold dilution series (100$0.4 \mathrm{n} M$ ), was injected at a flow rate of $20 \mu \mathrm{lmin}^{-1}$ for $240 \mathrm{~s}$, followed by a dissociation time of $900 \mathrm{~s}$. All binding experiments were performed at $25^{\circ} \mathrm{C}$ in $20 \mathrm{~m} M$ HEPES pH 7.4, $150 \mathrm{~m} M \mathrm{NaCl}, 0.005 \%(v / v)$ surfactant P20. BIAevaluation (GE Healthcare) and Origin 8 (OriginLab) were used to analyze and present the data. For a visual comparison of IgE-Fc binding curves to the different omalizumab constructs, the $100 \mathrm{n} M$ concentration for each was adjusted to give a maximal binding of 100 resonance units and these curves were overlaid.

\section{Results}

The nomenclature used for the omalizumab-derived Fabs and $\mathrm{scFv}$ reported here, and their crystal structures, is presented in Table 3. Heavy- and light-chain CDRs are defined as follows: CDRH1, Ser25-Asn36; CDRH2, Ser51-Asn59; CDRH3, Ala97-Val110; CDRL1, Arg24-Asn38; CDRL2, Tyr53-Ser60; CDRL3, Gln93-Thr101 (North et al., 2011).

3.1. Crystal structures of FabXol (wild-type omalizumab Fab): FabXol ${ }^{1}$ and FabXol ${ }^{2}$

The structure of FabXol (wild-type omalizumab Fab) was solved in two different crystal forms, which have also been reported by others (Jensen et al., 2015; Wright et al., 2015), and the space groups and unit-cell parameters of these structures, $\mathrm{FabXol}^{1}$ and $\mathrm{FabXol}^{2}$, the latter now reported at a substantially higher resolution, are provided in Table 1 . The structures reported here were the result of unsuccessful crystallization trials of the complex between FabXol and an unconstrained Fce3-4 molecule, but similar crystals were also grown from crystallization trials of FabXol in complex with IgE-Fc.

The FabXol ${ }^{1}$ structure (1.85 ̊ resolution) contains one Fab in the asymmetric unit, which forms two distinct interfaces with symmetry-related molecules (Fig. 1a). In the first interface, with an area of $\sim 395 \AA^{2}$, residues from all three heavychain $\mathrm{CDRs}$ contact $\mathrm{V}_{\mathrm{L}}$ and $\mathrm{C} \kappa$ domain framework residues from a symmetry-related molecule; namely, the $\mathrm{V}_{\mathrm{L}}$ domain 
$\mathrm{AB}, \mathrm{C}^{\prime \prime} \mathrm{D}$ and $\mathrm{EF}$ loops, and the $\mathrm{C} \kappa$ domain DE loop. In addition to van der Waals interactions, this interface comprises four hydrogen bonds, namely Thr30 (CDRH1)-Ser81 $\left(\mathrm{V}_{\mathrm{L}}\right)$, Ser31 (CDRH1)-Asp17 $\left(\mathrm{V}_{\mathrm{L}}\right)$, Tyr54 (CDRH2)-Arg65 $\left(\mathrm{V}_{\mathrm{L}}\right)$ and Tyr102 (CDRH3)-Ser175 (Cא) (Fig. 1b).

The second interface, with an area of $\sim 324 \AA^{2}$, includes an extensive network of hydrogen bonds between an edge $\beta$-strand from the $\mathrm{C} \gamma 1$ domain ( $\beta$-strand $\mathrm{G}$ ) and a short segment of $\beta$-strand structure in the $\mathrm{C} \kappa$ domain $\mathrm{CD}$ loop from a symmetry-related molecule. Here, the $\beta$-strands are arranged in a parallel manner, with hydrogen bonds between the main-chain atoms of Lys214-Lys218 (C $\gamma 1)$ and Leu158-
Ser160 (C $\kappa)$, and between the side chains of Lys217 (C $\gamma 1)$ and Ser160 (CK) (Fig. 1c). This interface is repeated throughout the crystal lattice, as an identical interface forms between

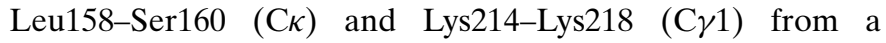
symmetry-related molecule.

The FabXol ${ }^{2}$ structure $(2.3 \AA$ resolution) contains two Fab molecules in the asymmetric unit, which are referred to here as $\mathrm{FabXol}^{2 A}$ and $\mathrm{FabXol}^{2 B}$. The CDRs of both molecules adopt similar conformations to those observed in the FabXol ${ }^{1}$ structure. CDRH1-3 residues also interact with the $\mathrm{V}_{\mathrm{L}}$ and $\mathrm{C} \kappa$ domain framework residues, akin to the first interface observed in the FabXol ${ }^{1}$ structure, which for $\mathrm{FabXol}^{2 B}$ also

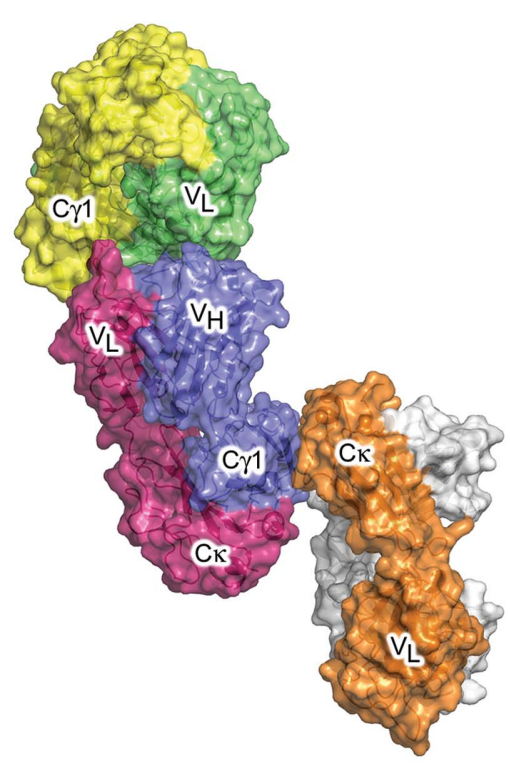

(a)

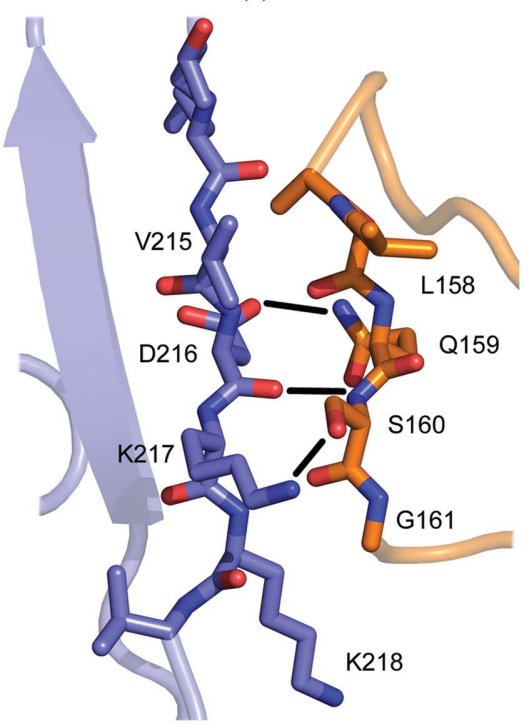

(c)

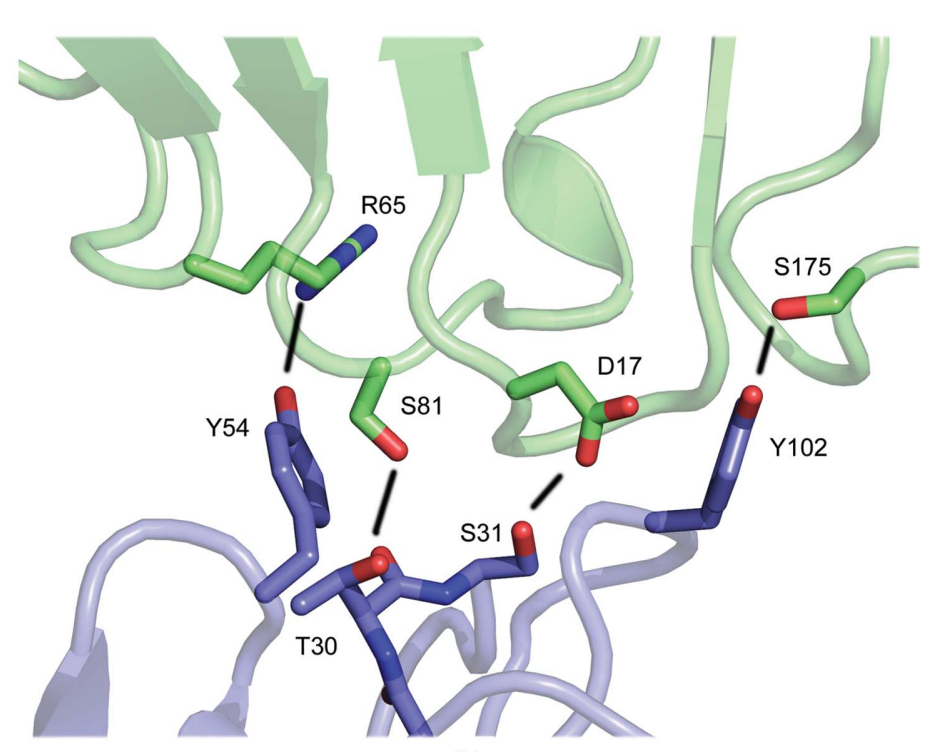

(b)

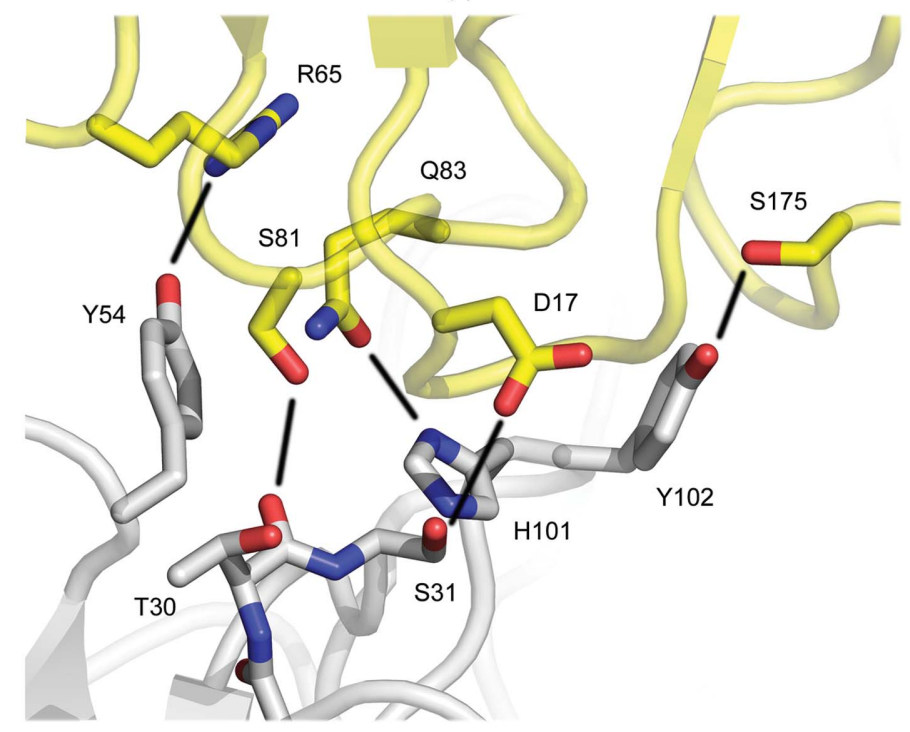

$(d)$

Figure 1

Structure of the omalizumab Fab (FabXol). (a) The FabXol ${ }^{1}$ structure contains one Fab molecule (pink and blue) in the asymmetric unit. The heavychain CDRs of this Fab contact the $\mathrm{V}_{\mathrm{L}}$ and $\mathrm{C} \kappa$ domains (the latter hidden in this view) of one symmetry-related molecule (green and yellow) and the $\mathrm{C} \kappa$ domain of another (orange and gray). (b) Interface between heavy-chain CDR residues (blue) and $\mathrm{V}_{\mathrm{L}}$ and $\mathrm{C} \kappa$ domain framework residues from a symmetry-related molecule (green) in the FabXol ${ }^{1}$ structure. Hydrogen bonds are depicted by black lines. ( $c$ ) Interface between an edge $\beta$-strand from the $\mathrm{C} \gamma 1$ domain (blue) and the $\mathrm{C} \kappa$ domain from a symmetry-related molecule (orange) in the FabXol ${ }^{1}$ structure. Hydrogen bonds are depicted by black lines. (d) Interface between heavy-chain CDR residues (gray) and $\mathrm{V}_{\mathrm{L}}$ and $\mathrm{C} \kappa$ domain framework residues from a symmetry-related molecule (yellow) for $\mathrm{FabXol}^{2 B}$, which includes a hydrogen bond between His101 (CDRH3) and $\mathrm{Gln} 81$ ( $\mathrm{V}_{\mathrm{L}}$ domain). Hydrogen bonds are depicted by black lines. 
includes a hydrogen bond between His101 (CDRH3) and $\mathrm{Gln} 83\left(\mathrm{~V}_{\mathrm{L}}\right)$ (Fig. 1d). The arrangement of Fabs in the FabXol ${ }^{2}$ asymmetric unit precludes the propagation of the second, $\beta$-strand-mediated interface throughout the crystal lattice by a single Fab molecule, as in the FabXol ${ }^{1}$ structure. However, interactions between $\mathrm{FabXol}^{2 A}$ and $\mathrm{FabXol}^{2 B}$, and different symmetry-related molecules, each display this same $\beta$-strand interaction, in which Lys214-Lys218 (C $\gamma 1)$ from $\mathrm{FabXol}^{2 \mathrm{~A}}$ interact with Leu158-Ser160 $(\mathrm{C} \kappa)$ from one symmetry-related molecule, while Leu158-Ser160 (C $\kappa)$ from $\mathrm{FabXol}^{2 B}$ interact with Lys214-Lys218 (C $\gamma 1)$ from a different symmetry-related molecule.

\subsection{Crystal structure of scFvXol (omalizumab-derived scFv)}

We also attempted to crystallize the complex between a single-chain form of omalizumab ( $\mathrm{scFvXol}$ ) and $\mathrm{IgE}-\mathrm{Fc}$, but were unsuccessful. However, we solved the crystal structure of $\mathrm{scFvXol}$ alone, in which the light- and heavy-chain variable domains are connected by a $\left(\mathrm{Gly}_{4} \mathrm{Ser}\right)_{4}$ linker, to $2.3 \AA$ resolution (Table 1). The scFvXol structure contains one molecule in the asymmetric unit.

In this structure, the $\beta$-strand-mediated crystal packing interaction observed in the $\mathrm{FabXol}^{1}$ and $\mathrm{FabXol}^{2}$ structures is absent, as the construct lacks the $\mathrm{C} \gamma 1$ and $\mathrm{C} \kappa$ domains. However, CDRH1-3 residues from a symmetry-related molecule contact the $\mathrm{V}_{\mathrm{L}}$ domain of scFvXol in a similar manner to the first interface described for the $\mathrm{FabXol}^{1}$ and $\mathrm{FabXol}^{2}$ structures, although the interface area is reduced from $\sim 395$ to $\sim 290 \AA^{2}$ due to the absence of the $\mathrm{C} \kappa$ domain in $\mathrm{scFvXol}$.

\subsection{Mutagenesis strategy I: disrupting the interaction between the $\mathrm{C} \gamma 1$ and $\mathrm{C} \kappa$ domains}

Crystallization trials of the complexes between FabXol (omalizumab Fab) and IgE-Fc, between scFvXol (omalizumab-derived scFv) and IgE-Fc, and between FabXol and an unconstrained Fce3-4 molecule all led to selective crystallization of the Fab or were unsuccessful. Two recurring interfaces in the Fab and scFvXol structures, described in Section 3.1, suggested a route to disrupt crystal packing interactions without mutating the CDR residues responsible for IgE-Fc binding.

We first attempted to disrupt the interface between the edge $\beta$-strand ( $\beta$-strand $\mathrm{G}$ ) from the $\mathrm{C} \gamma 1$ domain (Lys214-Lys218) and the short $\beta$-strand segment in the $\mathrm{C} \kappa$ domain $\mathrm{CD}$ loop (Leu158-Ser160), observed in the $\mathrm{FabXol}^{1}$ and $\mathrm{FabXol}^{2}$ structures. Leu158 from the $\mathrm{C} \kappa$ domain CD loop was mutated to proline, with the aim of altering its secondary structure, to disrupt the extensive, hydrogen-bond-mediated interactions. This omalizumab-derived Leu158Pro mutant Fab was termed FabXol1.

\subsection{Crystal structures of FabXol1 (omalizumab-derived} Leu158Pro mutant Fab): FabXol ${ }^{1}$ and FabXol ${ }^{2}$

The Leu158Pro mutation alone was not sufficient to prevent selective crystallization of the Fab, and the structures reported here were the result of unsuccessful crystallization trials of the complex between FabXol1 and IgE-Fc. Two structures were solved for FabXol1, in new crystal forms, and the space groups and unit-cell parameters of these structures, FabXol1 ${ }^{1}$ and FabXol1 ${ }^{2}$, are provided in Table 2.

The FabXol $1^{1}$ structure (1.8 ̊ resolution) contains two Fab molecules (FabXol $1^{1 A}$ and FabXol $1^{1 B}$ ) in the asymmetric unit (Fig. 2a). In this structure, the network of hydrogen bonds observed in the FabXol structures between $\beta$-strands of the $\mathrm{C} \gamma 1$ and $\mathrm{C} \kappa$ domains is indeed disrupted, but the engineered residue, Pro158, now forms other crystal packing interactions.

In molecule FabXol1 ${ }^{1 A}$, Asp155-Gln159, and His193 (C $\kappa$ ), including Pro158, form an interface with Pro62, Lys65-Arg67 and $\operatorname{Arg} 87\left(\mathrm{~V}_{\mathrm{H}}\right)$ from a crystallographic symmetry-related molecule, burying a surface area of $187 \AA^{2}$ (Fig. $2 b$ ). In molecule FabXol1 ${ }^{1 B}$, Lys149, Gln151, Lys153, Asn156, Pro158-Gly161, and Glu199 (C $\kappa)$, form an interface of $215 \AA^{2}$ with Gly161, Ser163 and Gln164 $(\mathrm{C} \kappa)$, Ala88 and Glu89 $\left(\mathrm{V}_{\mathrm{H}}\right)$, and Leu178-Gly182 $(\mathrm{C} \gamma 1)$ from the noncrystallographic symmetry-related molecule FabXol1 ${ }^{1 A}$ (Fig. $2 c$ ).

CDRH1-3 residues in both molecules of the FabXol $1^{1}$ structure adopt essentially identical conformations to those found in the FabXol ${ }^{1}$ and $\mathrm{FabXol}^{2}$ (wild-type omalizumab $\mathrm{Fab}$ ) and scFvXol (omalizumab-derived scFv) structures. They form similar crystal packing interactions to the first interface described for the FabXol ${ }^{1}$ structure, in which the heavy-chain CDRs contact the $\mathrm{V}_{\mathrm{L}}$ domain $\mathrm{AB}, \mathrm{C}^{\prime \prime} \mathrm{D}$ and $\mathrm{EF}$ loops, and the $\mathrm{C} \kappa$ domain DE loop from a symmetry-related molecule. In both molecules, hydrogen bonds form between Ser31 (CDRH1) and Asp17 $\left(\mathrm{V}_{\mathrm{L}}\right)$, between Tyr54 (CDRH2) and $\operatorname{Arg} 65\left(\mathrm{~V}_{\mathrm{L}}\right)$ and between Tyr102 (CDRH3) and Ser175 $(\mathrm{C} \kappa)$ (Fig. 2d).

The FabXol1 $1^{2}$ structure (2.5 A resolution) contains four Fab molecules (FabXol1 ${ }^{2 A}-$ FabXol1 ${ }^{2 D}$ ) in the asymmetric unit. In this structure, the packing environment of Pro158 differs from that in the FabXol1 ${ }^{1}$ structure. Again, the $\beta$-strand interactions between $\mathrm{C} \gamma 1$ and $\mathrm{C} \kappa$ domains are disrupted, but new packing interactions involving Pro158 are formed. In all four molecules of the FabXol1 ${ }^{2}$ structure, Pro158 forms van der Waals interactions with Pro158-Ser160 $(\mathrm{C} \kappa)$ from a noncrystallographic symmetry-related Fab (Fig. $3 a$ ). In this manner, Pro158 mediates light-chain/light-chain interactions between FabXol1 $^{2 A}$ and FabXol1 ${ }^{2 C}$, and between FabXol1 ${ }^{2 B}$ and FabXol1 ${ }^{2 D}$. Due to the arrangement of the four Fab molecules in the asymmetric unit, Pro158 from FabXol1 ${ }^{2 C}$ is positioned at an interface comprising three Fabs $\left(\mathrm{FabXol}^{2 A}-\mathrm{FabXo1}^{2 C}\right.$ ), and in addition to the interface with Pro158-Ser160 from FabXol1 ${ }^{2 A}$, also contacts Arg87 $\left(\mathrm{V}_{\mathrm{H}}\right)$ from FabXol1 ${ }^{2 B}$ (Fig. 3a).

In molecules FabXol1 ${ }^{2 A}$ and FabXol1 ${ }^{2 B}$, the heavy-chain CDRs adopt similar conformations to those in the FabXol, scFvXol and FabXol $1^{1}$ structures. CDR residues from FabXol1 ${ }^{2 B}$ form a similar interface with $\mathrm{V}_{\mathrm{L}}$ and $\mathrm{C} \kappa$ domain framework residues from a symmetry-related molecule; hydrogen bonds form between Ser31 (CDRH1) and Asp17 $\left(\mathrm{V}_{\mathrm{L}}\right)$, between $\operatorname{Tyr} 54(\mathrm{CDRH} 2)$ and $\operatorname{Arg} 65\left(\mathrm{~V}_{\mathrm{L}}\right)$, between His101 (CDRH3) and Gln83 $\left(\mathrm{V}_{\mathrm{L}}\right)$ and between Tyr102 (CDRH3) and Ser175 (C $)$, burying a surface area of $384 \AA^{2}$. 
Although FabXol1 ${ }^{2 A}$ contacts the $\mathrm{V}_{\mathrm{L}}$ and $\mathrm{C} \kappa$ domains of a symmetry-related molecule, the position of this molecule is shifted and the interface area, which is reduced to $274 \AA^{2}$, contains a single hydrogen bond between Tyr102 (CDRH3) and Asp174 (Cא) (Fig. 3b).

By contrast, the CDRH1 and CDRH3 conformations differ in molecules FabXol1 ${ }^{2 C}$ and FabXol1 ${ }^{2 D}$ compared with the other structures described thus far. In these molecules, binding of a glycerol molecule causes the Tyr33 (CDRH1) and His101 (CDRH3) side chains to adopt substantially different positions (Fig. 3c), the implications of which are discussed later. Crystal contacts for FabXol1 ${ }^{2 C}$ and FabXol ${ }^{2 D}$ also differ markedly compared with the other Fabs. In FabXol1 ${ }^{2 C}$, Thr30 and Ser31 (CDRH1) form hydrogen bonds with Thr73 and $\operatorname{Ser} 28\left(\mathrm{~V}_{\mathrm{L}}\right)$, respectively, from one symmetry-related molecule, while Tyr102 (CDRH3) packs against Gly15 and Gly16 $\left(\mathrm{V}_{\mathrm{H}}\right)$ from another molecule (Fig. 3d). On the other hand, in FabXol1 $^{2 D}$, only the interaction between Tyr102 and Gly15 and Gly16 from the second symmetry-related molecule is found; the first molecule is positioned further away, precluding hydrogen bonds between Thr30 (CDRH1) and Thr73, and between Ser31 (CDRH1) and Ser28. By contrast, CDRH2 residues do not participate in any crystal contacts, and adopt similar conformations to those in FabXol1 ${ }^{2 A}$ and FabXol1 ${ }^{2 B}$.

Despite the different contacts formed by CDRH1 and CDRH3 in molecules FabXol $1^{2 C}$ and FabXol ${ }^{2 D}$, the packing environment would not preclude the CDR conformations observed in the FabXol, scFvXol and FabXol $1^{1}$ structures, and in molecules FabXol1 ${ }^{2 A}$ and FabXol1 ${ }^{2 B}$.

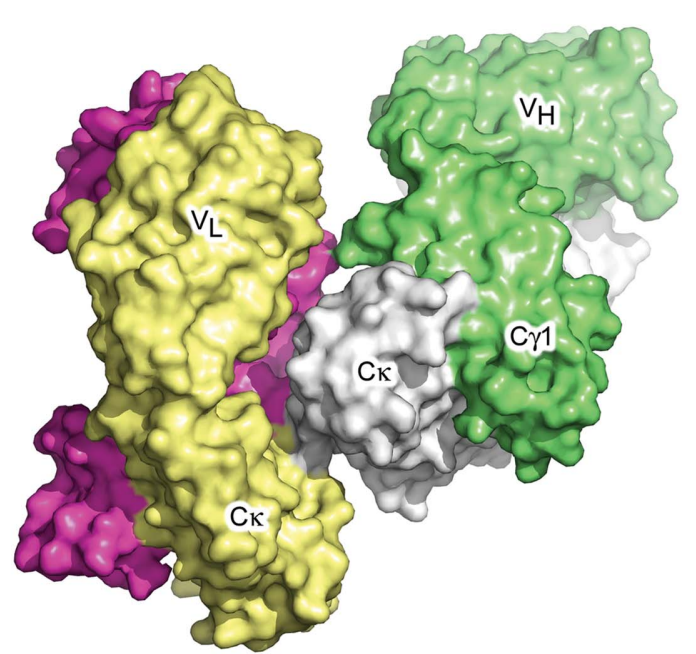

(a)

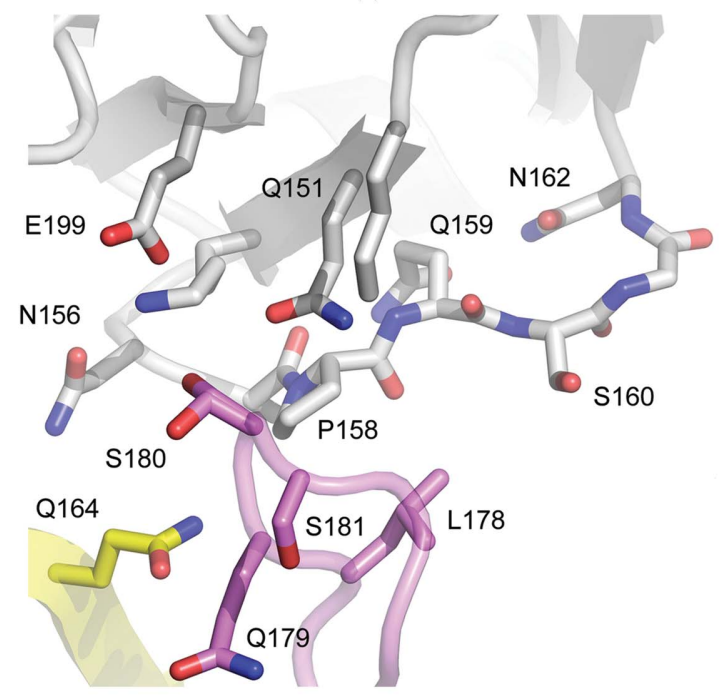

(c)

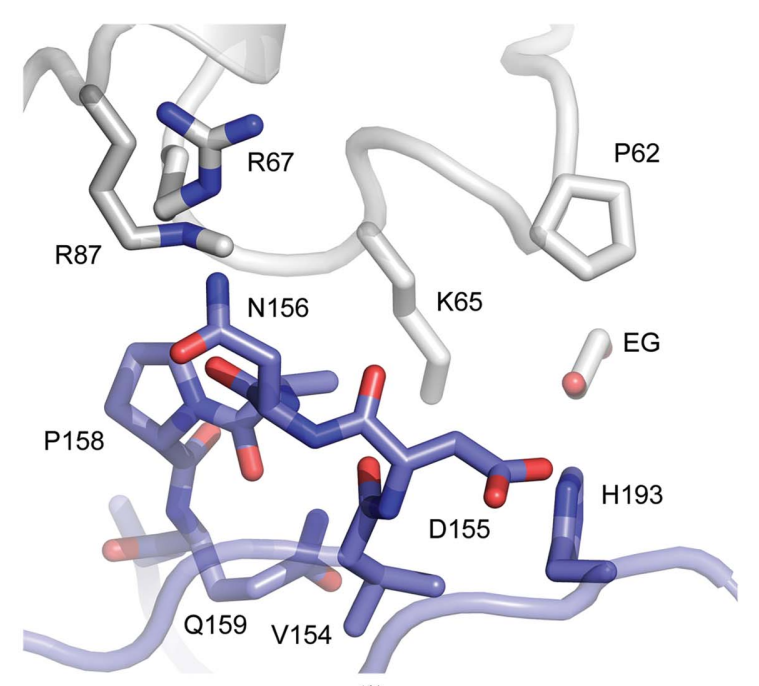

(b)

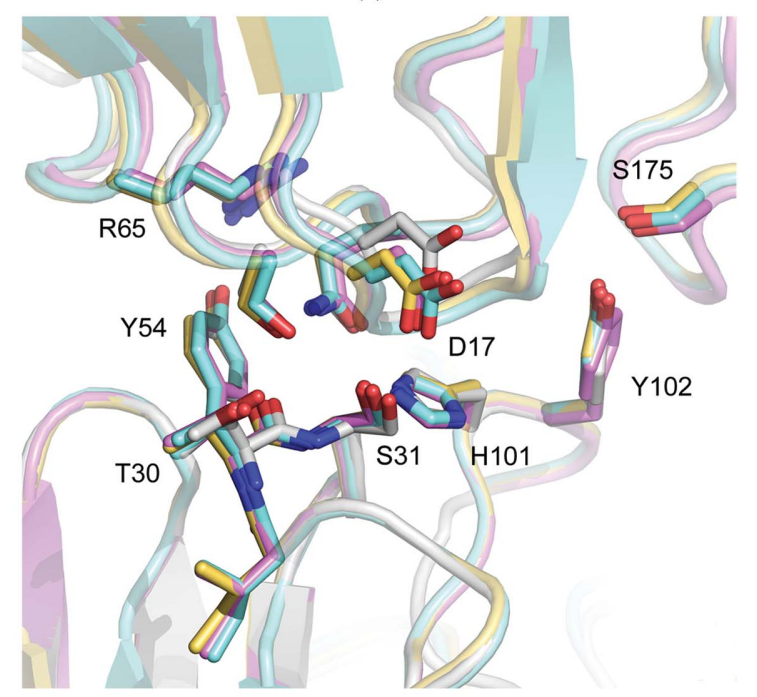

(d)

Figure 2

Structure FabXol $1^{1}$ of the omalizumab-derived Leu158Pro mutant (FabXol1). (a) The FabXol $1^{1}$ structure contains two molecules (pink and yellow/green and gray) in the asymmetric unit. (b) Interface between residues $155-159$ of the $\mathrm{C} \kappa$ domain (blue) of molecule FabXol1 ${ }^{1 A}$ and the $\mathrm{V}_{\mathrm{H}}$ domain of a symmetry-related molecule (gray). An ethylene glycol molecule (EG) is also bound at this interface. (c) Interface between the $\mathrm{C} \kappa$ domain (gray) of molecule FabXol $1^{1 B}$ and the $\mathrm{C} \kappa$ domain (yellow) and $\mathrm{C} \gamma 1$ domain (pink) of the noncrystallographic symmetry-related molecule, FabXol1 ${ }^{1 A}$. $(d)$ Conformations for the CDRH1-3 residues, and their crystal packing interactions with the $\mathrm{V}_{\mathrm{L}}$ domain (and $\mathrm{C} \kappa$ domain in the Fabs), are similar for $\mathrm{FabXol}^{1}$ (pink), FabXol1 ${ }^{1 A}$ (yellow), FabXol1 ${ }^{1 B}$ (blue) and scFvXol (gray). 
3.5. Mutagenesis strategy II: disrupting packing interactions involving the heavy-chain CDRs

Although the Leu158Pro mutation in the short $\beta$-strand segment of the $\mathrm{C} \kappa$ domain $\mathrm{CD}$ loop disrupted the interaction with the $\mathrm{C} \gamma 1$ domain edge $\beta$-strand (strand $\mathrm{G}$ ), it did not prevent selective crystallization of the Fab. We next attempted to disrupt the interface between the heavy-chain CDRs and the $\mathrm{V}_{\mathrm{L}}$ and $\mathrm{C} \kappa$ domain framework residues. As most of this interface involves interactions between the CDRs and the $\mathrm{V}_{\mathrm{L}}$ domain, and mutating the CDRs could adversely affect the interaction with IgE-Fc, we mutated Ser81 and Gln83 from the $\mathrm{V}_{\mathrm{L}}$ domain EF loop, which contribute to this interface, to Arg81 and Arg83, respectively, thus incorporating bulkier, charged side chains. We created two omalizumab-derived Fabs, namely FabXol2, with Ser81Arg and Gln83Arg muta- tions, and FabXol3, which additionally contains a Leu158Pro mutation. Thermal stability measurements revealed that the incorporation of these three point mutations, either alone or in combination with one another, did not substantially affect the overall stability of the Fabs (Table 4).

\subsection{Crystal structures of FabXol2 (omalizumab-derived}

Ser81Arg, GIn83Arg mutant Fab) and FabXol3 (omalizumabderived Ser81Arg, Gln83Arg, Leu158Pro mutant Fab)

Complexes between IgE-Fc and both of the omalizumabderived Fabs that contained the Ser81Arg and Gln83Arg mutations were eventually crystallized. Crystals with a similar morphology were grown for each complex, although the FabXol3/IgE-Fc complex crystals diffracted to higher

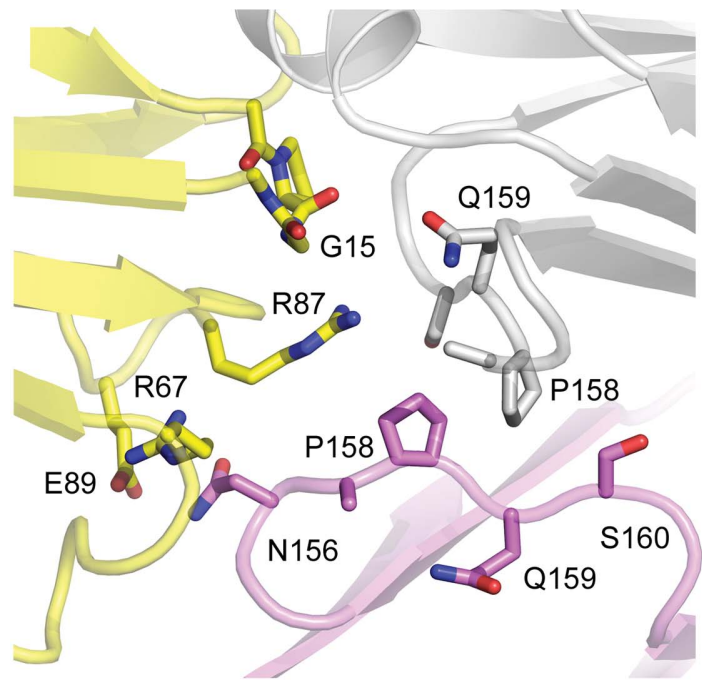

(a)

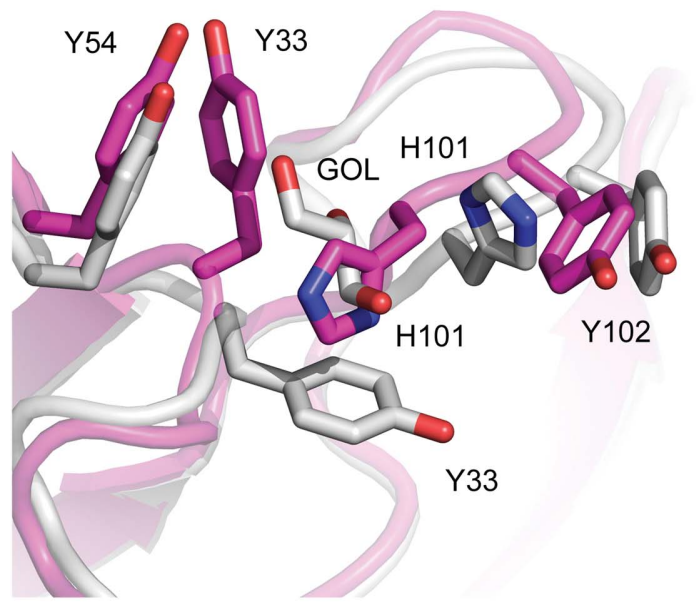

(c)

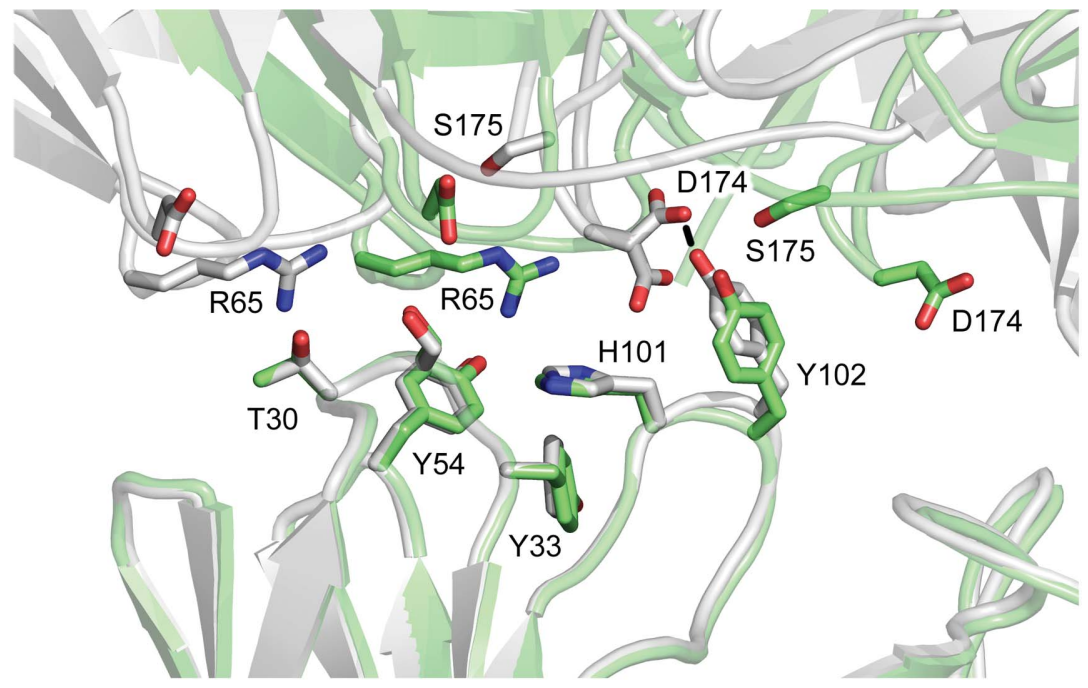

(b)
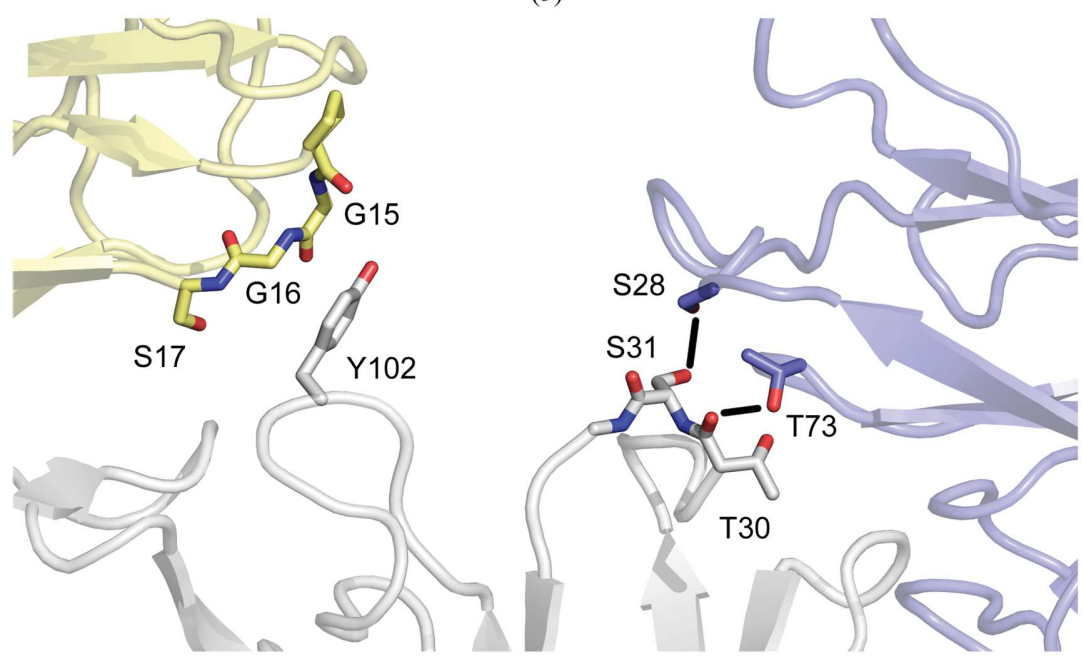

(d)

Figure 3

Structure FabXol1 ${ }^{2}$ of the omalizumab-derived Leu158Pro mutant (FabXol1). (a) In FabXol1 ${ }^{2 C}$ (pink), residues Pro158-Ser160 form an interface with the $C \kappa$ domain from FabXol1 ${ }^{2 A}$ (gray) and the $\mathrm{V}_{\mathrm{H}}$ domain from FabXol1 ${ }^{2 B}$ (yellow). (b) The FabXol ${ }^{2 A}$ (gray) and FabXol1 ${ }^{2 B}$ (green) CDRs adopt similar conformations, and both contact the $\mathrm{V}_{\mathrm{L}}$ and $\mathrm{C} \kappa$ domains from a symmetry-related molecule. A shift in the position of the symmetry-related molecule relative to FabXol1 ${ }^{2 A}$ reduces the interface area, and only a single hydrogen bond is formed between Tyr102 (CDRH3) and Asp174 (C $\kappa$ domain). (c) Binding of a glycerol molecule (GOL) in FabXol1 ${ }^{2 C}$ (gray) causes the Tyr33 and His101 side chains to adopt substantially different positions compared with those in FabXol1 (pink). (d) In FabXol1 ${ }^{2 C}$ (gray), Thr30 and Ser31 form hydrogen bonds with Thr73 and Ser28, respectively, from a symmetry-related molecule (blue). Tyr102 packs against Gly15 and Gly16 from a different symmetry-related molecule (yellow). 
Table 4

Thermal stabilities of the omalizumab-derived Fabs.

\begin{tabular}{ll}
\hline & $T_{\mathrm{m}}\left({ }^{\circ} \mathrm{C}\right)$ \\
\hline FabXol & $79.9 \pm 0.5$ \\
FabXol1 & $79.0 \pm 0.7$ \\
FabXol2 & $77.1 \pm 0.5$ \\
FabXol3 & $78.8 \pm 0.4$ \\
\hline
\end{tabular}

resolution, and we recently reported the crystal structure of the complex to $3.7 \AA$ resolution (Davies et al., 2017).

To understand the effects of the Ser81Arg and Gln83Arg $\left(\mathrm{V}_{\mathrm{L}}\right)$ mutations on Fab crystal packing interactions, we solved the structures of FabXol2 and FabXol3 alone. Both FabXol2 and FabXol3 crystallized in the same crystal form (Table 2), with one Fab molecule in the asymmetric unit. With the exception of the light-chain residue 158, which is leucine in FabXol2 and proline in FabXol3, the structures are otherwise essentially identical.

The packing interactions that involve $\mathrm{V}_{\mathrm{L}}$ domain residues 81 and 83 in the FabXol and FabXol1 structures are substantially different in the FabXol 2 and FabXol3 structures. In contrast to Ser81, which contacts Ser31 (CDRH1) and Tyr54 (CDRH2), Arg81 instead forms hydrogen bonds with Asn156 ( $\kappa \kappa$, symmetry-related molecule) (Fig. 4a). In FabXol3, Arg81 contacts Pro158 $(\mathrm{C} \kappa)$, while Leu158 is partially disordered in FabXol2. Furthermore, and in contrast to Gln83, which contacts Tyr33 (CDRH1), Tyr54 (CDRH2) and His101 (CDRH3) in the FabXol and FabXol1 structures, Arg83 does not participate in any crystal packing interactions in the FabXol2 and FabXol3 structures (Fig. 4a). As the overall structures of FabXol2 and FabXol3 are similar, further discussion will be limited to the FabXol3 structure, which was solved at higher resolution ( $1.45 \AA$ for FabXol3 compared with $2.05 \AA$ for FabXol2).

In the FabXol3 structure, CDRH1 and CDRH3 residues contact the $\mathrm{V}_{\mathrm{L}}$ domain of one symmetry-related molecule at an interface that includes hydrogen bonds between Ser31 (CDRH1) and Ser69, between Tyr27 (CDRH1) and Tyr57, between Tyr27 and Asp34, between Ser100 (CDRH3) and Asp30, between Phe103 (CDRH3, main chain) and Thr73, and between Gly104 (CDRH3, main chain) and Asp74 (Fig. 4b). On the other hand, Asp55 (CDRH2) forms a salt bridge with Lys211 from the $\mathrm{C} \kappa$ domain of a different symmetry-related Fab, and together with Gly56 (CDRH2) packs against Pro117 and Ser118 (Fig. 4c).

The FabXol3 CDRH1 and CDRH3 conformations are markedly different to those in the FabXol, scFvXol and FabXol1 structures; the nature and implications of these conformational differences are discussed later.

3.7. Conformational diversity in the CDRs: comparison of unbound and bound Fab structures

In the FabXol, scFvXol and FabXol $1^{1}$ structures, and in the molecules FabXol1 ${ }^{2 A}$ and FabXol1 ${ }^{2 B}$, the heavy-chain CDRs adopt similar conformations (Figs. $1 b, 1 d, 2 d$ and $3 b$ ). However, substantial conformational diversity is observed for CDRH1 and CDRH3 in molecules FabXol1 ${ }^{2 C}$ and FabXol1 ${ }^{2 D}$, and in FabXol3.

In molecules FabXol1 ${ }^{2 C}$ and FabXol1 ${ }^{2 D}$, a glycerol molecule occupies a structurally equivalent position to Ser378 and

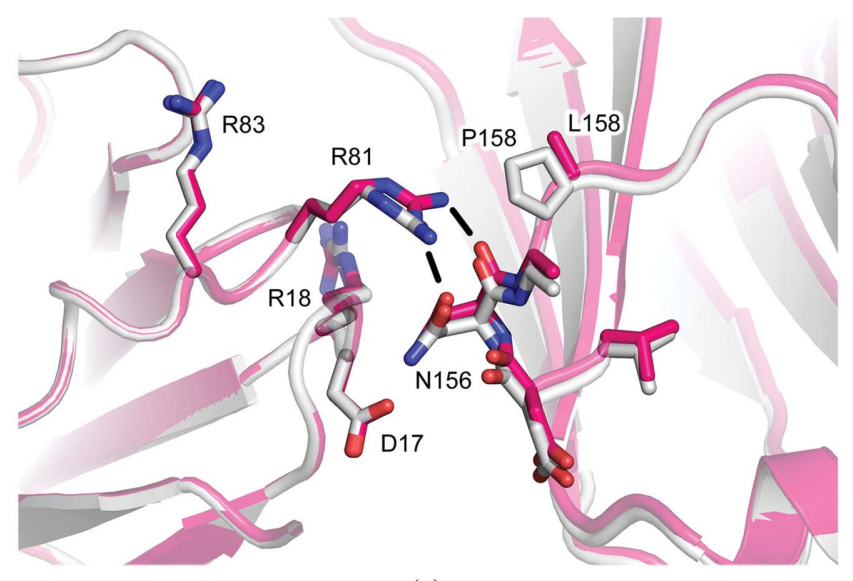

(a)

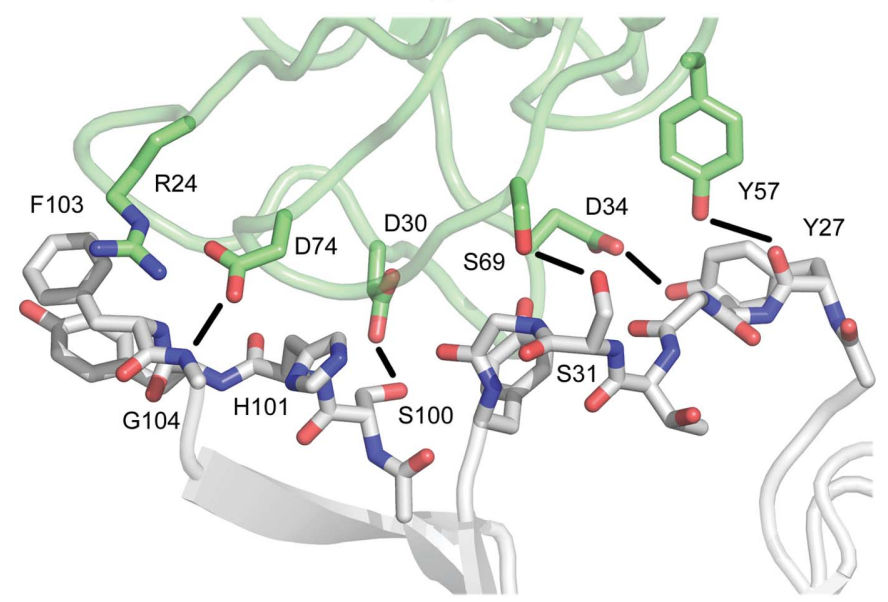

(b)

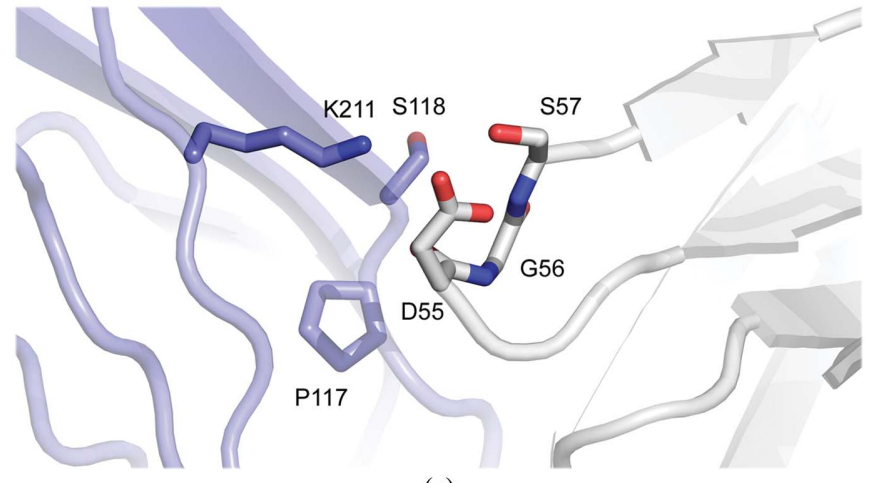

(c)

Figure 4

Structure of the omalizumab-derived Ser81Arg, Gln83Arg, Leu158Pro mutant (FabXol3). (a) In the FabXol3 (gray) and FabXol2 (pink) structures, Arg81 forms hydrogen bonds with Asn156. In the FabXol3 structure, Arg81 contacts Pro158, while Leu158 is partially disordered in the FabXol2 structure. Arg83 does not form any crystal packing interactions. (b) In the FabXol3 structure, CDRH1 and CDRH3 residues (gray) contact the $\mathrm{V}_{\mathrm{L}}$ domain of a symmetry-related molecule (green). Hydrogen bonds are depicted by black lines. (c) Asp55 (CDRH2) forms a salt bridge with Lys211 from the $\mathrm{C} \kappa$ domain of a symmetry-related molecule (blue). 
Gly379 from the CE3 domain in the complex between the omalizumab-derived Fab and IgE-Fc (Davies et al., 2017), altering the position of Tyr33 (CDRH1), which adopts a similar position to that in the IgE-Fc-bound Fab (Fig. 5a). The conformations of Ser31 (CDRH1) and Gly32 (CDRH1) are also similar to those in the complex, presumably due to the conformational change involving Tyr33. In the complex with IgE-Fc, Gly32 and Tyr33 from CDRH1 contribute to the interface with the $\mathrm{C} \varepsilon 3$ domain, packing against Ala377 and Ser378. The glycerol molecule, close to Tyr33, also causes the His101 (CDRH3) side chain to adopt a different position (Fig. 5a); however, the overall conformation of CDRH3 is otherwise similar to that in the unbound FabXol, scFvXol and FabXol1 ${ }^{1}$ structures and in the molecules FabXol1 ${ }^{2 A}$ and FabXol1 ${ }^{2 B}$.

In FabXol3, residues Ser25-Gly32 (CDRH1) adopt a markedly different conformation compared with the other unbound and bound Fab structures, which alters the positions of Tyr27 and Ile29; the Phe79 side chain, adjacent to CDRH1, also adopts a different position (Fig. $5 b$ ). On the other hand, Tyr33 adopts a similar position to that in the FabXol $1^{2 C}$ and FabXol1 ${ }^{2 D}$ molecules and the bound Fab structures. Comparison of the FabXol3 structure with the structure of the complex with IgE-Fc (Davies et al., 2017) reveals that the positions adopted by Ser25-Ser31, and Tyr33 in FabXol3 would not preclude an interaction with the $\mathrm{C} \varepsilon 3$ domain; however, Gly32 would clash with Ser378. This particular CDRH1 conformation thus appears to be incompatible with IgE binding. By contrast, in FabXol3, CDRH3 adopts a strikingly different conformation compared with the other Fab structures reported here (Fig. 5c). In these Fab structures, the CDRH3 conformation is incompatible with IgE binding due to steric clashes with the $\mathrm{C} \varepsilon 3$ domain. However, the CDRH3 conformation in the unbound FabXol3 structure is similar to

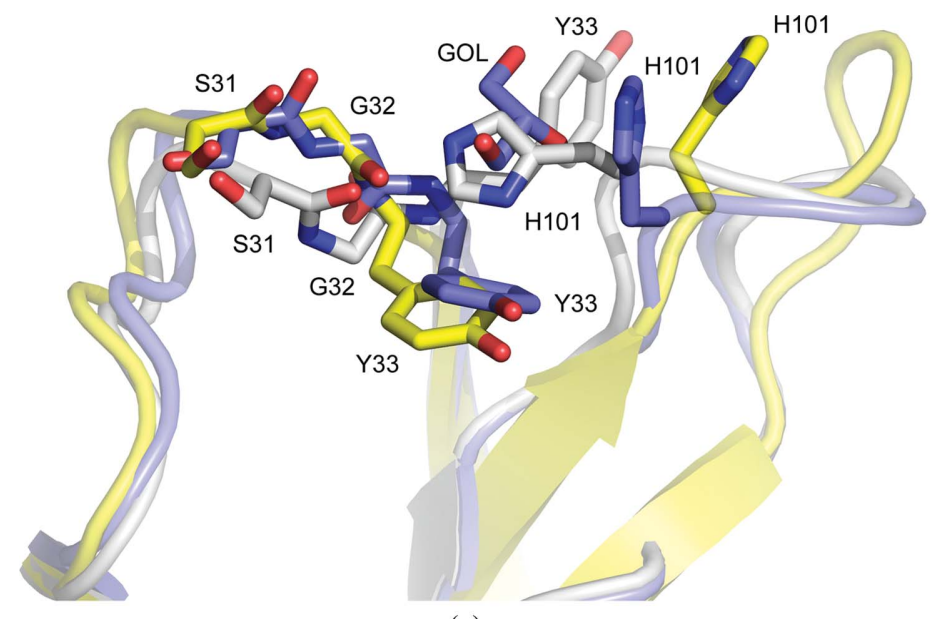

(a)

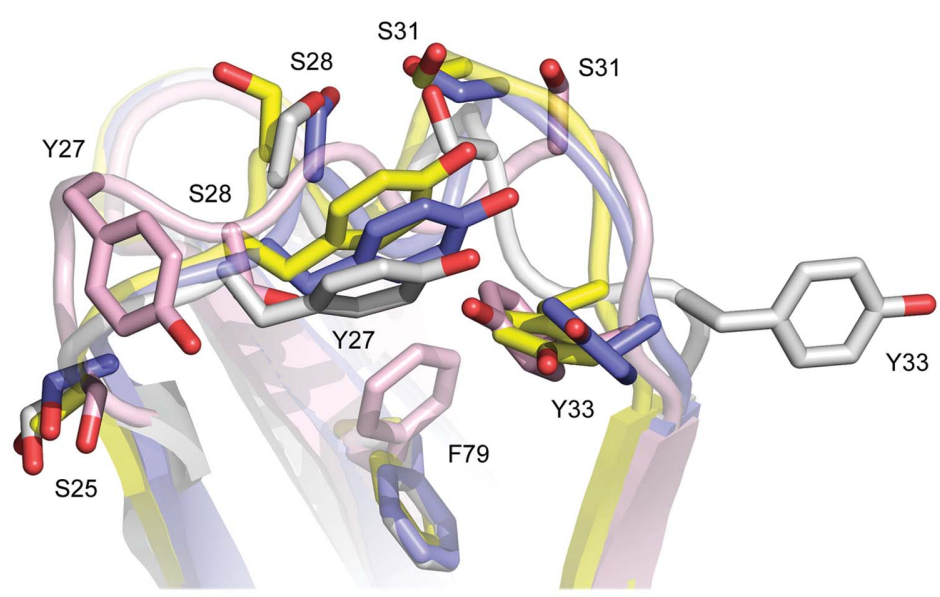

(b)

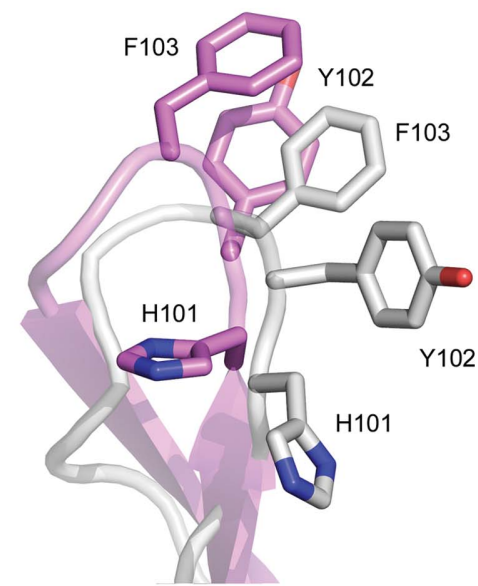

(c)

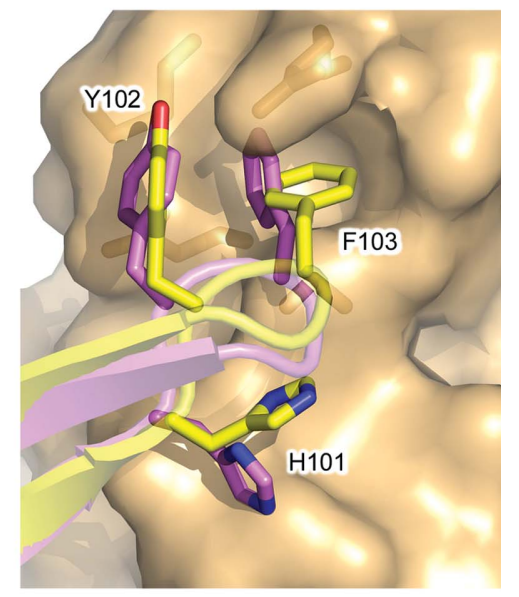

(d)

Figure 5

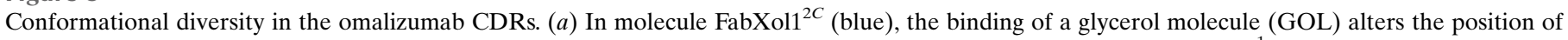

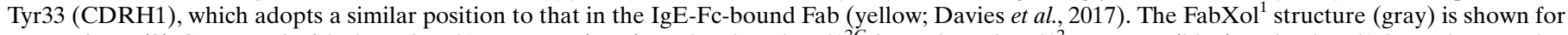

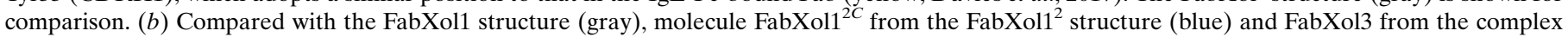

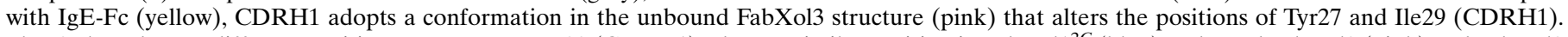

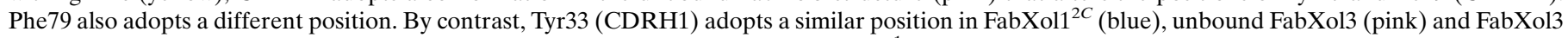

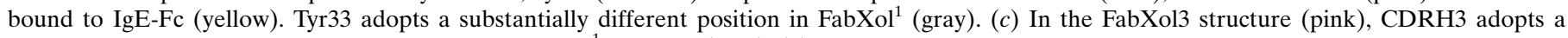

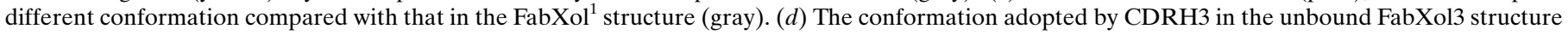

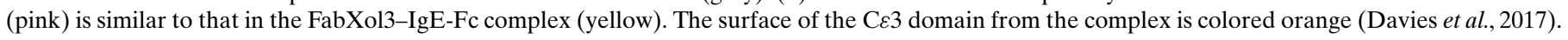


Table 5

Kinetics of omalizumab, the omalizumab-derived Fabs and scFv binding to IgE-Fc.

\begin{tabular}{lllll}
\hline $\begin{array}{l}\text { Molecule } \\
\text { immobilized }\end{array}$ & $k_{\text {on1 }}\left(M^{-1} \mathrm{~s}^{-1}\right)$ & $k_{\text {on2 }}\left(M^{-1} \mathrm{~s}^{-1}\right)$ & $k_{\text {off1 }}\left(\mathrm{s}^{-1}\right)$ & $k_{\text {off2 }}\left(\mathrm{s}^{-1}\right)$ \\
\hline Omalizumab & $3.3 \times 10^{5}$ & $2.9 \times 10^{5}$ & $7.0 \times 10^{-4}$ & $1.2 \times 10^{-2}$ \\
FabXol & $5.7 \times 10^{5}$ & $4.4 \times 10^{5}$ & $5.6 \times 10^{-4}$ & $1.2 \times 10^{-2}$ \\
FabXol2 & $5.1 \times 10^{5}$ & $3.3 \times 10^{5}$ & $4.5 \times 10^{-4}$ & $1.1 \times 10^{-2}$ \\
FabXol3 & $9.7 \times 10^{5}$ & $2.7 \times 10^{5}$ & $3.3 \times 10^{-4}$ & $9.0 \times 10^{-3}$ \\
scFvXol & $6.9 \times 10^{5}$ & $3.1 \times 10^{5}$ & $2.9 \times 10^{-4}$ & $8.7 \times 10^{-3}$ \\
\hline
\end{tabular}

the conformation adopted by CDRH3 in the FabXol3/IgE-Fc complex (Davies et al., 2017; Fig. 5d); a conformational change in the CDRH3 main chain causes a dramatic rearrangement in the positions of side-chain residues, particularly His101, Tyr102 and Phe103, which contact the C 83 domain in the complex.

In contrast to the structural diversity displayed by CDRH1 and $\mathrm{CDRH} 3$, the conformation of $\mathrm{CDRH} 2$ is conserved in the unbound $\mathrm{Fab}$ and $\mathrm{scFv}$ structures, and in the complexes of the omalizumab Fab with the constrained Fce3-4 molecule (Pennington et al., 2016) and of FabXol3 with IgE-Fc (Davies et al., 2017). Like CDRH2, the light-chain CDR conformations are also conserved; similar conformations are adopted in the 12 independent views reported here and in other unbound Fab structures (Jensen et al., 2015; Wright et al., 2015), which are similar to those in the complexes between the omalizumab Fab and the constrained Fce3-4 molecule (Pennington et al., 2016) and between FabXol3 and IgE-Fc (Davies et al., 2017). Nevertheless, the FabXol2 and FabXol3 crystal structures show substantial conformational diversity in the heavy-chain CDRs, and together with the FabXol1 ${ }^{2}$ structure reveal how conformations compatible with IgE binding are adopted in the unbound Fab.

3.8. Interaction of the omalizumab-derived Fabs and scFv with IgE-Fc in solution

The aim of our mutagenesis strategy was to disrupt the crystal packing interactions observed in the wild-type omalizumab (FabXol) crystal structures, without mutating the CDR residues responsible for $\mathrm{IgE}-\mathrm{Fc}$ binding and significantly affecting the affinity for IgE-Fc. We have previously demonstrated that the kinetics of the interaction between omalizumab and IgE-Fc are biphasic, with one high-affinity $(\sim 1 \mathrm{n} M)$ and one lower-affinity $(\sim 30 \mathrm{n} M)$ interaction (Davies et al., 2017), and that FabXol3 has a slightly higher affinity for IgE-Fc than FabXol (wild-type omalizumab Fab) and intact omalizumab (Davies et al., 2017).

We used surface plasmon resonance analysis to characterize further the interaction between IgE-Fc and the omalizumabderived Fab and $\mathrm{scFv}$ constructs. As we have shown previously, at the highest concentration tested $(100 \mathrm{n} M$ IgE-Fc), the omalizumab-derived Fabs and scFv all display the same mode of interaction with $\mathrm{IgE}-\mathrm{Fc}$, i.e. a biphasic model with one higher-affinity and one lower-affinity binding interaction (Davies et al., 2017). When these data were normalized to have the same maximum binding values, it was found that the association rates were similar to those for intact omalizumab (Davies et al., 2017; Table 5). However, a statistically significant trend of increasingly slower dissociation rates was observed: the dissociation rate for the omalizumab-derived Fab (FabXol) is slower than that for intact omalizumab, FabXol2 has a slower dissociation rate than FabXol and FabXol3 is even slower, while the scFvXol dissociation rate is the slowest of all (Table 5 and Supplementary Fig. S1).

\section{Discussion}

After unsuccessful attempts to crystallize the complex between the Fab fragment of the therapeutic anti-IgE omalizumab and IgE-Fc, and the Fce3-4 region, we designed a mutagenesis strategy to disrupt the substantial, and recurring, crystal packing interactions observed in different omalizumab Fab structures. We targeted crystal packing interactions at two different interfaces. The first interface comprised hydrogen bonds between an edge $\beta$-strand from the $\mathrm{C} \gamma 1$ domain ( $\beta$-strand G; Lys214-Lys218) and a short segment of $\beta$-strand structure in the $\mathrm{C} \kappa$ domain CD loop (Leu158-Ser160). The second interface involved the omalizumab heavy-chain CDRs and $\mathrm{V}_{\mathrm{L}}$ domain $\mathrm{AB}, \mathrm{C}^{\prime \prime} \mathrm{D}$ and $\mathrm{EF}$ loops and $\mathrm{C} \kappa$ domain $\mathrm{DE}$ loop. Our mutations were designed to disrupt these packing interactions without significantly affecting the affinity of omalizumab for $\mathrm{IgE}$, and as such were distal to the antigenbinding CDRs.

Packing interactions similar to that between the $\mathrm{C} \gamma 1$ domain edge $\beta$-strand (strand $\mathrm{G}$ ) and the $\mathrm{C} \kappa$ domain $\mathrm{CD}$ loop are found in a number of other crystal structures containing Fab fragments (see, for example, Hall et al., 2016; Lee et al., 2017; Li et al., 2009; Sickmier et al., 2016). Indeed, a variety of packing interactions involving hydrogen-bond networks between $\beta$-strands have been detected in crystal structures of intact antibodies and their fragments (Edmundson et al., 1999; Wingren et al., 2003), including antiparallel arrangements between edge strands in $\mathrm{C} \lambda$ and $\mathrm{C} \gamma 1$ domains (see, for example, Faber et al., 1998), $\mathrm{V}_{\mathrm{H}}$ domains (see, for example, Harris et al., 1998) and $\mathrm{V}_{\mathrm{L}}$ domains (see, for example, Bourne et al., 2002).

We mutated Leu158 from the omalizumab $\mathrm{C} \kappa$ domain $\mathrm{CD}$ loop to proline (omalizumab-derived mutant FabXol1) to disrupt the interface with strand $\mathrm{G}$ from the $\mathrm{C} \gamma 1$ domain, and although this was achieved, the FabXol1 molecule still crystallized preferentially, in different packing arrangements stabilized in part by the presence of Pro158.

We next targeted the crystal packing interactions between the omalizumab CDRs and $V_{L}$ and $C \kappa$ domain framework residues $\left(\mathrm{V}_{\mathrm{L}}\right.$ domain $\mathrm{AB}, \mathrm{C}^{\prime \prime} \mathrm{D}$ and $\mathrm{EF}$ loops and $\mathrm{C} \kappa$ domain DE loop) from symmetry-related molecules. We mutated Ser81 and Gln83 from the omalizumab $V_{L}$ domain EF loop to arginine and created two omalizumab-derived mutants: FabXol2 contained the Ser81Arg and Gln83Arg mutations, while FabXol3 additionally contained the Leu158Pro mutation. The IgE-Fc protein was successfully crystallized in complex with both FabXol2 and FabXol3, and the $3.7 \AA$ resolution crystal structure of the FabXol3/IgE-Fc complex 
was recently reported (Davies et al., 2017). Engineering the Ser81Arg and Gln83Arg mutations in the $V_{L}$ domain of the omalizumab Fab clearly disrupted the interactions seen in the FabXol structure, but these residues also formed new packing interactions in the FabXol2 and FabXol3 structures that were seen when these molecules were crystallized alone. Presumably, however, these packing contacts were collectively weaker than those in either FabXol or FabXol1, since they were unable to compete with the pre-formed Fab/IgE-Fc complexes and their crystallization.

Unbound IgE-Fc adopts an acutely bent conformation, in which the $\mathrm{C} \varepsilon 2$ domains fold back against the $\mathrm{F} c \varepsilon 3-4$ region (Doré et al., 2017; Holdom et al., 2011; Wan et al., 2002). IgE-Fc is more acutely bent in the crystal structure of the $\operatorname{sFc} \varepsilon \mathrm{RI} \alpha /$ IgE-Fc complex (Holdom et al., 2011), less acutely bent when in complex with sCD23 (Dhaliwal et al., 2017), partially bent when in complex with FabXol3 (Davies et al., 2017) and fully extended in the complexes with the anti-IgE Fabs a $\varepsilon \mathrm{Fab}$ and 8D6 (Chen et al., 2018; Drinkwater et al., 2014); these structures demonstrate that $\mathrm{IgE}-\mathrm{Fc}$ is conformationally dynamic. However, despite this flexibility, IgE adopts a predominantly bent conformation in solution (Beavil et al., 1995; Davis et al., 1990; Holowka \& Baird, 1983; Holowka et al., 1985; Hunt et al., 2012; Zheng et al., 1991, 1992). The propensity for IgE-Fc to adopt such a bent conformation might account for the selective crystallization of the omalizumab Fab and the omalizumab-derived mutant FabXol1. Bending of IgE-Fc, from the partially bent conformation observed in the FabXol3/IgE-Fc complex to the acutely bent structure, would disrupt one of the omalizumab binding sites on the $\mathrm{C} \varepsilon 3$ domain. In the FabXol3/ IgE-Fc complex, Arg81 and Arg83 from one FabXol3 molecule contact one of the $\mathrm{C} \varepsilon 2$ domains, in addition to the omalizumab binding site on the $\mathrm{C} \varepsilon 3$ domain. This additional interaction might stabilize the partially bent conformation in the complex.

In IgE-Fc and $\mathrm{Fc} \varepsilon 3-4$, the $\mathrm{C} \varepsilon 3$ domains adopt a range of conformations relative to one another, from closed to open (Chen et al., 2018; Cohen et al., 2014; Davies et al., 2017; Dhaliwal et al., 2012, 2014, 2017; Doré et al., 2017; Drinkwater et al., 2014; Garman et al., 2000; Holdom et al., 2011; Jabs et al., 2018; Wan et al., 2002; Wurzburg \& Jardetzky, 2009; Wurzburg et al., 2000; Yuan et al., 2013); this conformational diversity is crucial for the allosteric regulation of $\mathrm{IgE}$ binding to its receptors, FceRI and CD23 (Borthakur et al., 2012; Dhaliwal et al., 2012). The flexibility of the $\mathrm{C} \varepsilon 3$ domains could account for our failure to crystallize the complex between the omalizumab Fab and the unconstrained Fce3-4 molecule, which lacks the $\mathrm{C} \varepsilon 2$ domains. Notably, the reported omalizumab Fab complex (Pennington et al., 2016) is with an Fce3-4 molecule that contains an engineered disulfide bond, which locks the $\mathrm{C} \varepsilon 3$ domains into a closed conformation, thus reducing the overall flexibility of the complex.

Fab fragments are invaluable tools as chaperone proteins for crystallization, and are used for their ability to trap different conformations or reduce flexibility in the target protein (Bukowska \& Grütter, 2013; Griffin \& Lawson, 2011; Rasmussen et al., 2007; Sun et al., 2018; Tamura et al., 2019;
Uysal et al., 2009). However, in our case, crystallization trials of our conformationally flexible target protein, IgE-Fc, in complex with the Fab fragment of the therapeutic anti- $\operatorname{IgE}$ antibody omalizumab resulted in the disruption of pre-formed complexes and selective crystallization of the Fab alone.

Here, we have described a successful mutagenesis strategy in which framework regions of the omalizumab Fab were engineered to disrupt recurring crystal packing interactions in the Fab crystal structures, without significantly altering the stability of the Fab, nor its affinity for IgE-Fc. Although disrupting the hydrogen-bond-mediated interactions between $\beta$-strands did not prevent selective crystallization of the Fab, the recurring interface between the light chain and CDRs was disrupted by introducing bulkier residues through point mutations in the light-chain framework regions.

This approach, of introducing point mutations distal to the antigen-binding CDRs to disrupt undesired crystal packing interactions, could assist in the structure determination of Fabs in complex either with similarly conformationally flexible, or indeed inflexible, target proteins.

\section{Acknowledgements}

We thank Dr Anthony Keeble for helpful discussions. We thank Marty Rajaratnam (King's College London) for use of the in-house X-ray facility, and Diamond Light Source (Harwell, UK) for access to beamlines I02, I03, I04, I04-1 and I24 (proposal Nos. MX1220, MX7656 and MX9495) that contributed to the results presented here. We acknowledge the support of the Centre for Biomolecular Spectroscopy, King's College London. We thank Hanna Hailu for protein expression and Sue Cross, Sarfaraj Topia and Vidisha Krishnan for protein purification. TC, JTH and AJH are employees of UCB Celltech. TC and AJH are in receipt of stock options. A patent application has been filed based on elements of this work.

\section{Funding information}

The following funding is acknowledged: Medical Research Council (grant No. G1100090 to Andrew J. Beavil, James M. McDonnell and Brian J. Sutton); Wellcome Trust (award No. 085944 to James M. McDonnell and Brian J. Sutton).

\section{References}

Beavil, A. J., Young, R. J., Sutton, B. J. \& Perkins, S. J. (1995). Biochemistry, 34, 14449-14461.

Borthakur, S., Hibbert, R. G., Pang, M. O., Yahya, N., Bax, H. J., Kao, M. W., Cooper, A. M., Beavil, A. J., Sutton, B. J., Gould, H. J. \& McDonnell, J. M. (2012). J. Biol. Chem. 287, 31457-31461.

Bourne, P. C., Ramsland, P. A., Shan, L., Fan, Z.-C., DeWitt, C. R., Shultz, B. B., Terzyan, S. S., Moomaw, C. R., Slaughter, C. A., Guddat, L. W. \& Edmundson, A. B. (2002). Acta Cryst. D58, 815823.

Bukowska, M. A. \& Grütter, M. G. (2013). Curr. Opin. Struct. Biol. 23, 409-416.

Chen, J.-B., Ramadani, F., Pang, M. O. Y., Beavil, R. L., Holdom, M. D., Mitropoulou, A. N., Beavil, A. J., Gould, H. J., Chang, T. W., Sutton, B. J., McDonnell, J. M. \& Davies, A. M. (2018). Sci. Rep. 8, 11548. 
Chen, V. B., Arendall, W. B., Headd, J. J., Keedy, D. A., Immormino, R. M., Kapral, G. J., Murray, L. W., Richardson, J. S. \& Richardson, D. C. (2010). Acta Cryst. D66, 12-21.

Cohen, E. S., Dobson, C. L., Käck, H., Wang, B., Sims, D. A., Lloyd, C. O., England, E., Rees, D. G., Guo, H., Karagiannis, S. N., O'Brien, S., Persdotter, S., Ekdahl, H., Butler, R., Keyes, F., Oakley, S., Carlsson, M., Briend, E., Wilkinson, T., Anderson, I. K., Monk, P. D., von Wachenfeldt, K., Eriksson, P. O., Gould, H. J., Vaughan, T. J. \& May, R. D. (2014). $m A b s, 6,755-763$.

Davies, A. M., Allan, E. G., Keeble, A. H., Delgado, J., Cossins, B. P., Mitropoulou, A. N., Pang, M. O. Y., Ceska, T., Beavil, A. J., Craggs, G., Westwood, M., Henry, A. J., McDonnell, J. M. \& Sutton, B. J. (2017). J. Biol. Chem. 292, 9975-9987.

Davis, K. G., Glennie, M., Harding, S. E. \& Burton, D. R. (1990). Biochem. Soc. Trans. 18, 935-936.

Dhaliwal, B., Pang, M. O. Y., Keeble, A. H., James, L. K., Gould, H. J., McDonnell, J. M., Sutton, B. J. \& Beavil, A. J. (2017). Sci. Rep. 7, 45533.

Dhaliwal, B., Pang, M. O. Y., Yuan, D., Beavil, A. J. \& Sutton, B. J. (2014). Acta Cryst. F70, 305-309.

Dhaliwal, B., Yuan, D., Pang, M. O., Henry, A. J., Cain, K., Oxbrow, A., Fabiane, S. M., Beavil, A. J., McDonnell, J. M., Gould, H. J. \& Sutton, B. J. (2012). Proc. Natl Acad. Sci. USA, 109, 12686-12691.

Doré, K. A., Davies, A. M., Drinkwater, N., Beavil, A. J., McDonnell, J. M. \& Sutton, B. J. (2017). Biochim. Biophys. Acta, 1865, 13361347.

Drinkwater, N., Cossins, B. P., Keeble, A. H., Wright, M., Cain, K., Hailu, H., Oxbrow, A., Delgado, J., Shuttleworth, L. K., Kao, M. W.-P., McDonnell, J. M., Beavil, A. J., Henry, A. J. \& Sutton, B. J. (2014). Nat. Struct. Mol. Biol. 21, 397-404.

Edmundson, A. B., DeWitt, C. R., Goldsteen, B. Z. \& Ramsland, P. A. (1999). J. Cryst. Growth, 196, 276-284.

Emsley, P., Lohkamp, B., Scott, W. G. \& Cowtan, K. (2010). Acta Cryst. D66, 486-501.

Evans, P. (2006). Acta Cryst. D62, 72-82.

Evans, P. R. \& Murshudov, G. N. (2013). Acta Cryst. D69, 1204-1214.

Faber, C., Shan, L., Fan, Z., Guddat, L. W., Furebring, C., Ohlin, M., Borrebaeck, C. A. K. \& Edmundson, A. B. (1998). Immunotechnology, 3, 253-270.

Fuh, G., Wu, P., Liang, W.-C., Ultsch, M., Lee, C. V., Moffat, B. \& Wiesmann, C. (2006). J. Biol. Chem. 281, 6625-6631.

Garman, S. C., Wurzburg, B. A., Tarchevskaya, S. S., Kinet, J.-P. \& Jardetzky, T. S. (2000). Nature, 406, 259-266.

Gould, H. J. \& Sutton, B. J. (2008). Nat. Rev. Immunol. 8, 205-217.

Griffin, L. \& Lawson, A. (2011). Clin. Exp. Immunol. 165, 285291.

Hall, G., Cullen, E., Sawmynaden, K., Arnold, J., Fox, S., Cowan, R., Muskett, F. W., Matthews, D., Merritt, A., Kettleborough, C., Cruikshank, W., Taylor, D., Bayliss, R. \& Carr, M. D. (2016). J. Biol. Chem. 291, 16840-16848.

Harris, L. J., Skaletsky, E. \& McPherson, A. (1998). J. Mol. Biol. 275, 861-872.

Holdom, M. D., Davies, A. M., Nettleship, J. E., Bagby, S. C., Dhaliwal, B., Girardi, E., Hunt, J., Gould, H. J., Beavil, A. J., McDonnell, J. M., Owens, R. J. \& Sutton, B. J. (2011). Nat. Struct. Mol. Biol. 18, 571-576.

Holgate, S., Casale, T., Wenzel, S., Bousquet, J., Deniz, Y. \& Reisner, C. (2005). J. Allergy Clin. Immunol. 115, 459-465.

Holgate, S. T. (2014). World Allergy Organ. J. 7, 17.

Holowka, D. \& Baird, B. (1983). Biochemistry, 22, 3475-3484.

Holowka, D., Conrad, D. H. \& Baird, B. (1985). Biochemistry, 24, 6260-6267.

Hunt, J., Keeble, A. H., Dale, R. E., Corbett, M. K., Beavil, R. L., Levitt, J., Swann, M. J., Suhling, K., Ameer-Beg, S., Sutton, B. J. \& Beavil, A. J. (2012). J. Biol. Chem. 287, 17459-17470.

Jabs, F., Plum, M., Laursen, N. S., Jensen, R. K., Mølgaard, B., Miehe, M., Mandolesi, M., Rauber, M. M., Pfützner, W., Jakob, T., Möbs, C., Andersen, G. R. \& Spillner, E. (2018). Nat. Commun. 9, 7.
Jensen, R. K., Plum, M., Tjerrild, L., Jakob, T., Spillner, E. \& Andersen, G. R. (2015). Acta Cryst. F71, 419-426.

Kabsch, W. (2010). Acta Cryst. D66, 125-132.

Krissinel, E. \& Henrick, K. (2007). J. Mol. Biol. 372, 774-797.

Lee, J. U., Shin, W., Son, J. Y., Yoo, K.-Y. \& Heo, Y.-S. (2017). Int. J. Mol. Sci. 18, 228.

Leslie, A. G. W. \& Powell, H. R. (2007). Evolving Methods for Macromolecular Crystallography, edited by R. Read \& J. Sussman, pp. 41-51. Dordrecht: Springer.

Li, S., Wang, H., Peng, B., Zhang, M., Zhang, D., Hou, S., Guo, Y. \& Ding, J. (2009). Proc. Natl Acad. Sci. USA, 106, 4349-4354.

Liebschner, D., Afonine, P. V., Baker, M. L., Bunkóczi, G., Chen, V. B., Croll, T. I., Hintze, B., Hung, L.-W., Jain, S., McCoy, A. J., Moriarty, N. W., Oeffner, R. D., Poon, B. K., Prisant, M. G., Read, R. J., Richardson, J. S., Richardson, D. C., Sammito, M. D., Sobolev, O. V., Stockwell, D. H., Terwilliger, T. C., Urzhumtsev, A. G., Videau, L. L., Williams, C. J. \& Adams, P. D. (2019). Acta Cryst. D75, 861-877.

McCoy, A. J., Grosse-Kunstleve, R. W., Adams, P. D., Winn, M. D., Storoni, L. C. \& Read, R. J. (2007). J. Appl. Cryst. 40, 658674.

Murshudov, G. N., Skubák, P., Lebedev, A. A., Pannu, N. S., Steiner, R. A., Nicholls, R. A., Winn, M. D., Long, F. \& Vagin, A. A. (2011). Acta Cryst. D67, 355-367.

North, B., Lehmann, A. \& Dunbrack, R. L. (2011). J. Mol. Biol. 406, 228-256.

Pennington, L. F., Tarchevskaya, S., Brigger, D., Sathiyamoorthy, K., Graham, M. T., Nadeau, K. C., Eggel, A. \& Jardetzky, T. S. (2016). Nat. Commun. 7, 11610.

Rasmussen, S. G. F., Choi, H.-J., Rosenbaum, D. M., Kobilka, T. S., Thian, F. S., Edwards, P. C., Burghammer, M., Ratnala, V. R. P., Sanishvili, R., Fischetti, R. F., Schertler, G. F. X., Weis, W. I. \& Kobilka, B. K. (2007). Nature, 450, 383-387.

Sickmier, E. A., Kurzeja, R. J., Michelsen, K., Vazir, M., Yang, E. \& Tasker, A. S. (2016). PLoS One, 11, e0163366.

Sun, J., Paduch, M., Kim, S. A., Kramer, R. M., Barrios, A. F., Lu, V., Luke, J., Usatyuk, S., Kossiakoff, A. A. \& Tan, S. (2018). Proc. Natl Acad. Sci. USA, 115, 10010-10015.

Sussman, G., Hébert, J., Barron, C., Bian, J., Caron-Guay, R. M., Laflamme, S. \& Stern, S. (2014). Ann. Allergy Asthma Immunol. 112, 170-174.

Tamura, R., Oi, R., Akashi, S., Kaneko, M. K., Kato, Y. \& Nogi, T. (2019). Protein Sci. 28, 823-836.

Uysal, S., Vásquez, V., Tereshko, V., Esaki, K., Fellouse, F. A., Sidhu, S. S., Koide, S., Perozo, E. \& Kossiakoff, A. (2009). Proc. Natl Acad. Sci. USA, 106, 6644-6649.

Vagin, A. \& Teplyakov, A. (2010). Acta Cryst. D66, 22-25.

Wan, T., Beavil, R. L., Fabiane, S. M., Beavil, A. J., Sohi, M. K., Keown, M., Young, R. J., Henry, A. J., Owens, R. J., Gould, H. J. \& Sutton, B. J. (2002). Nat. Immunol. 3, 681-686.

Weatherill, E. E., Cain, K. L., Heywood, S. P., Compson, J. E., Heads, J. T., Adams, R. \& Humphreys, D. P. (2012). Protein Eng. Des. Sel. 25, 321-329.

Wingren, C., Edmundson, A. B. \& Borrebaeck, C. A. (2003). Protein Eng. 16, 255-264.

Winn, M. D., Ballard, C. C., Cowtan, K. D., Dodson, E. J., Emsley, P., Evans, P. R., Keegan, R. M., Krissinel, E. B., Leslie, A. G. W., McCoy, A., McNicholas, S. J., Murshudov, G. N., Pannu, N. S., Potterton, E. A., Powell, H. R., Read, R. J., Vagin, A. \& Wilson, K. S. (2011). Acta Cryst. D67, 235-242.

Winter, G. (2010). J. Appl. Cryst. 43, 186-190.

Wright, J. D., Chu, H.-M., Huang, C.-H., Ma, C., Chang, T. W. \& Lim, C. (2015). Sci. Rep. 5, 11581.

Wurzburg, B. A., Garman, S. C. \& Jardetzky, T. S. (2000). Immunity, 13, 375-385.

Wurzburg, B. A. \& Jardetzky, T. S. (2009). J. Mol. Biol. 393, 176190.

Young, R. J., Owens, R. J., Mackay, G. A., Chan, C. M., Shi, J., Hide, 
M., Francis, D. M., Henry, A. J., Sutton, B. J. \& Gould, H. J. (1995). Protein Eng. Des. Sel. 8, 193-199.

Yuan, D., Keeble, A. H., Hibbert, R. G., Fabiane, S., Gould, H. J., McDonnell, J. M., Beavil, A. J., Sutton, B. J. \& Dhaliwal, B. (2013). J. Biol. Chem. 288, 21667-21677.

Zheng, L., Li, B., Qian, W., Zhao, L., Cao, Z., Shi, S., Gao, J., Zhang,
D., Hou, S., Dai, J., Wang, H. \& Guo, Y. (2008). Biochem. Biophys. Res. Commun. 375, 619-622.

Zheng, Y., Shopes, B., Holowka, D. \& Baird, B. (1991). Biochemistry, 30, 9125-9132.

Zheng, Y., Shopes, B., Holowka, D. \& Baird, B. (1992). Biochemistry, 31, 7446-7456. 